\title{
ESTUDO SOBRE A EICONAL DO ESPALHAMENTO ELÁSTICO PION-PRÓTON
} A ALTAS ENERGIAS

\author{
ANTÔNIO BRAZ DE PÁDUA ${ }^{1}$ \\ JORGE DIAS DE DEUS ${ }^{2}$
}

PÁDUA, A.B. de; DIASDE DEUS, J. Estudo sobre a eiconal do espalhamento elástico pion-próton a altas energias. Semina: Ci. Exatas/Tecnológicas, Londrina, v. 16, n. 4, p. 516-528, dez. 1995.

RESUMO: Analisamos os espalhamentos elásticos $\pi^{ \pm} p$ a $50 \mathrm{GeV} / \mathrm{c}$ e 200GeV/c através do formalismo do parâmetro de impacto e de forma independente de modelo teórico. Os dados experimentais de seção de choque diferencial elástica, no intervalo de momento transferido $\left.10 \leq-t \leq 10(\mathrm{GeV} / \mathrm{c})^{2}\right]_{1}$ foram ajustados numericamente supondo-se uma amplitude de espalhamento elástico puramente imaginária e expressa como uma soma de exponenciais em-t. A parte real da amplitude de espalhamento foi incorporada via argumentos de relaçöes de dispersão. A partir dos ajustes, calculamos a eiconal e testamos as hipóteses da fatoraçào e do "geometrical scaling". Nossas resultados sugerem uma eiconal que tem os dois comportamentos juntos, ou seja: na regiáo central, ela é aproximadamente fatorávele, na regiăo periférica, seu comportamento é consistente com o "geometrical scaling".

PALAVRAS-CHAVE: Espalhamento Elástico, Altas Energias, Pion, Próton, Seção de Choque Diferencial Elástica, Amplitude de Espalhamento Elástico, Parâmetro de Impacto, Eiconal, Fatoração e "Geometrical Scaling".

\section{INTRODUÇÃO}

A investigação sistemática dos processos de espalhamento hadrón-hádron a altas energias, que se iniciou em meados dos anos cinqüenta, teve um extraordinário avanço a partir do final da década de sessenta com a construção de grandes aceleradores no CERN (EUROPA), no FERMILAB (USA) e em SERPUKHOV (RÚSSIA).

Os resultados experimentais obtidos das colisóes hadrônicas realizadas nesses aceleradores revelam que a maioria das reaçōes que ocorrem são inelásticas, com $\sigma_{\mathrm{e} i} / \sigma_{\mathrm{tot}}$ variando entre $15 \%$ e $25 \%$ (GIACOMELLI ${ }^{20}$; PADUA ${ }^{26}$ ).

Muito embora o espalhamento elástico ocorra com menor probabilidade num processo hádron-hádron, seus dados experimentais desempenham um papel muito importante para a compreensão de alguns aspectos básicos das interações fortes. Por exemplo, os dados experimentais de seção de choque diferencial elástica nos permite investigar, entre outras coisas, a forma e tamanho da distribuição de matéria hadrônica das partículas interagentes e também, como estas grandezas se comportam com relação à variação da energia. Um outro aspecto fundamental do espalhamento elástico é que no espaço do parâmetro de impacto, a condição de unitaridade (GIACOMELLI' ${ }^{20}$ ), inter-relaciona as seções de choque totai, inelástica e elástica. Com isto, podemos obter algumas informações sobre a componente inelástica a partir do conhecimento dos dados experimentais do espalhamento elástico.

Em razão das dificuldades de se obter uma teoria geral para os processos hadrônicos, uma parte significativa dos estudos teóricos procura descrever algumas propriedades fenomenológicas das colisões elásticas utilizando-se parametrizações de dados experimentais.

O estudo feito através da parametrizações é independente de modelo teórico. Normalmente para realizálo, ajustam-se numericamente os dados experimentais da seção de choque diferenciai elástica, escolhendo expressões adequadas para a amplitude de espalhamento elástico em função do momento transferido, ou até mesmo, da energia. Neste trabalho, adotando a sistemática de parametrizações, apresentamos uma análise das reaçōes $\pi^{*} p$ e $\pi p$ no espaço do parâmetro de impacto, feita a partir dos dados experimentais nas energias $s^{1 / 2}=9.78 \mathrm{GeV}\left(\mathrm{p}_{\mathrm{tab}}=50 \mathrm{GeV} / \mathrm{c}\right) \mathrm{e}$ $s^{1 / 2}=19.42 \mathrm{GeV}\left(p_{\text {lab }}=200 \mathrm{GeV} / \mathrm{c}\right)$ e no intervalo de momento transferido: $0 \leq-t \leq 10(\mathrm{GeV} / \mathrm{c})^{2}$.

Os dados experimentais de seção de choque diferencial elástica foram parametrizados supondo-se que a amplitude de espalhamento elástico é imaginária e que tem um zero na posição do $1^{\circ}$. mínimo ("dip"), ou seja, ela troca de sinal e permanece contínua quando

1 - Departamento de Física - CCE - Universidade Estadual de Londrina - Caixa Postal 6001, Londrina, PR, Brasil, CEP 86051-970.

2 - Departamento de Física - Instituto Superior Técnico - Av. Rovisco Pais 1096, Lisboa Codex, Portugal. 
passa por este ponto. A parte real da amplitude de espalhamento que, ao ser considerada, preenche o vazio deixado pela parte imaginária na região do $1^{\circ}$. mínimo, foi incluída através da Fórmula de Martin (MARTIN ${ }^{25}$ ). A partir dos parâmetros de ajuste e da representação do parâmetro de impacto, calculamos a eiconal e analisamos seu comportamento em função da energia e do parâmetro de impacto.

Desde a formulação do modelo eiconal de ChouYang em 1968 (CHOU \& YANG ${ }^{14}$ ), vários trabalhos $\left(\mathrm{CHOU}^{12}\right.$; $\mathrm{CHOU}^{13}$; FRANÇA \& HAMA ${ }^{19}$; AMALDI \& SCHUBERT ${ }^{4}$; FEARNLEY ${ }^{18}$; BELLANDI et $\mathrm{al}^{8}$; PÁDUA ${ }^{26}$, etc.) foram feitos com o objetivo de descobrir o comportamento da eiconal com relação ã energia e ao parâmetro de impacto. Como se sabe, a eiconal está diretamente relacionada com o potencial de interação $\left(\mathrm{PERL}^{27}\right.$; GLAUBER ${ }^{21}$ ) e que portanto, um conhecimento seguro sobre ela pode fornecer subsídios mais confiáveis para testar os diferentes modelos teóricos sobre interações fortes e, também, mostrar um caminho de como incorporar corretamente a dependência energética nos modelos ótico-geométricos. Esses últimos, exceto pelos problemas com relação à incorporação da energia, têm tido muito sucesso na descrição das estruturas complicadas apresentadas pelos dados experimentais de seção de choque diferencial elástica das colisões hadrônicas a altas energias.

A maioria dos estudos fenomenolõgicos sobre a eiconal foi realizada a partir da reação $\mathrm{pp}$. Isto ocorreu porque esta reação possui as medidas mais precisas e os dados experimentais mais completos e abundantes dentre as principais reações hádron-hádron. Quanto a $p \bar{p}$, somente apos a construçāo do Spps - COLLIDER no CERN no início dos anos oiterta, é que se tornou objeto de intenso estudo. Embora os dados experimentais de $p \bar{p}$ sejam incompletos e de baixa estatistica, quando comparados com pp, eles têm uma grande vantagem "sobre estes, ou seja, foram obtidos a energias muito mais altas. Afualmente, chegou-se a $s^{1 / 2}=$ $1800 \mathrm{GeV}$ no TEVATRON dO FERMILAB.

No caso das reaçōes méson-próton, a situação não é muito estimulante para estudos fenomenológicos a altas energias. Alèm da escassez de dados experimentais, as máximas energias para as quais se tēm medidas são extremamente baixas quando compara.

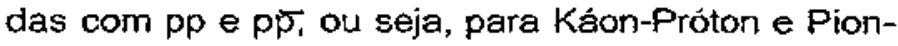
Próton existem dados experimentais até $\left.\mathrm{s}^{1 / 2}\right)_{\max }$ $20 \mathrm{GeV}$, enquanto que no caso de $\left.\mathrm{pp}, \mathrm{s}^{1 / 2}\right)_{\max } \sim 60 \mathrm{GeV} \mathrm{e}$ de $\left.p \bar{p}, s^{12}\right)_{\text {masx }} \sim 2000 \mathrm{GeV}$.

Em razão dessa limitação de energia, nas reações méson-próton existem poucos trabaihos dedicados ao cálculo da eiconal e funções correlatas para essas interações. No caso de Káon-Próton, destacamos um trabalho nosso (COVOLAN et $a !^{15}$ ), em que fizemos uma extensa análise na representação do parâmetro de impacto e de forma independente de modelo. Quanto ao caso do Pion-Próton, desconhecemos a existência de algum estudo que permita uma comparação direta e mais completa com os resultados existentes de $p p, p \bar{p}, K^{*} p$ e K"p.

Desta forma, proponos, neste trabalho, fazer uma investigação mais cuidadosa das reaçōes $\pi * p$ e $\pi p$ utilizando-se o formalismo do parâmetro de impacto e comparar os resultados obtidos com aqueles das reaçōes acima mencionadas.

\section{DISCUSSÃO TEÓRICA}

A seção de choque diferencial elástica está ligada ã amplitude de espalhamento elástico através da relação

$$
\frac{d \sigma}{d t}=\pi|f(s, t)|^{z}
$$

De forma geral, $f(s, t)$ é uma função complexa, ou seja,

$$
f(S, t)=\operatorname{Ref}(s, t)+i a(s, t),
$$

onde $\mathrm{a}(\mathrm{s}, \mathrm{t})=\operatorname{Imf}(\mathrm{s}, \mathrm{t})$.

A altas energias, os dados experimentais do espalhamento elástico hádron-hádron revelam que este processo é, em primeira aproximação, basicamente difrativo (ALBERS \& GOGGí ${ }^{2}$; KAMRAN ${ }^{23}$; CASTALDI \& SANGUINETTI ${ }^{11}$ ) e que, portanto, pode ser descrito por uma amplitude de espalhamento puramente imaginária com $\operatorname{Ref}(s, t)=0$. Assim, podemos escrever

$$
\frac{\mathrm{d} \sigma}{\mathrm{dt}}=\pi|\mathrm{a}(\mathrm{s}, \mathrm{t})|^{2} \text {. }
$$

O formalismo do parâmetro de impacto relaciona a amplitude de espalhamento elástico com a distribuição de matéria hadrônica das partículas interagentes através de uma Transformada de Fourier-Bessel bidimensional.

Os modelos eiconais $\left\langle\mathrm{CHOU} \&\right.$ YANG $^{14}$; HAYOT \& SUKHATME ${ }^{22}$ ), os quais adotam este formalismo, tem como ponto básico a análise da eiconal $\chi(s, b)$. No espaço do parâmetro de impacto, a eiconal está relacionada com amplitude de espalhamento elástico pela expressāo

$$
f(s, t)=i \int_{0}^{\infty}\left(1-e^{i x(s . b)}\right) J_{0}(b \sqrt{-t}) b d b
$$


Em geral $\chi(s, b)$ é uma função complexa, ou seja,

$$
\chi(s, b)=\chi_{R}(s, b)+i \Omega(s, b) .
$$

onde $\chi_{R}(s, b)=\operatorname{Re\chi }(s, b)$ e $\left.\Omega(s, b)=\operatorname{lm} \chi s, b\right)$. A funçăo $\Omega(\mathrm{s}, \mathrm{b})$ é conhecida como opacidade.

Substituindo-se (5) em (4) e usando-se (2) obtemos as seguintes relações

$$
\operatorname{Ref}(s, t)=\int_{0}^{\infty} \operatorname{sen} \chi_{R}(s, b) e^{-\Omega(s, b)} J_{0}(b \sqrt{-t}) b d b .
$$

e

$$
a(s, t)=\int_{0}^{\infty}\left[1-\cos \chi_{R}(s, b) e^{-\Omega(s, 0)}\right] J_{0}(b \sqrt{-t}) b d b .
$$

Se o espalhamento for puramente difrativo teremos $\chi_{R}(s, b)=0$. Consequentemente, Ref $(s, t)=0$ e

$$
a(s, t)=\int_{0}^{\infty}\left[1-e^{-s(s, b)}\right] J_{0}(b \sqrt{-t}) b c b .
$$

Finalmente, substituindo-se (8) em (3), obtemos a expressão usual que relaciona a seção de choque diferencial elástica à eicona! (agora igual a opacidade) através da representação do parâmetro de impacto, para colisões hádron-hádron puramente difrativas e no regime de altas energias,

$$
\frac{d \sigma}{d t}=\pi\left[\int_{0}^{\infty}\left(1-e^{-\Omega(s . b)}\right) J_{0}(b \sqrt{-t}) b d b\right]^{2} .
$$

\section{AJUSTE DOS DADOS EXPERIMENTAIS}

Para a realização dos ajustes, necessitamos dos dados experimentais da seçäo de choque diferencial elástica $(\mathrm{d} \sigma / \mathrm{dt})\left[\mathrm{mb} /(\mathrm{GeV} / \mathrm{c})^{2}\right] \times(-t)\left[(\mathrm{GeV} / \mathrm{c})^{2}\right]$ e da razäo entre a parte real e a parte imaginária da amplitude de espalhamento elástico para frente $p(s, 0) \times s^{1 / 2}[\mathrm{GeV}]$ (dacui para frente a chamaremos de $\rho$ ).

Os conjuntos de dados mais completos para as reações $\pi^{*} p$ foram obtidos nas energias $p_{1 a b 0}=50 \mathrm{GeV} / \mathrm{c}$ $\left(s^{1 / 2}=9.78 \mathrm{GeV}\right)$ e $p_{\text {lad }}=200 \mathrm{GeV} / c\left(s^{1 / 2}=19.42 \mathrm{GeV}\right)$ nos aceleradores do CERN e do FERMILAB.

Este trabalho será desenvolvido com base nestas energias, pois além de apresentarem os melhores dados existentes para as reaçōes pion-próton, elas são aquelas que permitem fazer uma comparação mais direta com os resultados existentes para $K^{+} p, K p, p p e$ $\mathrm{p} \overline{\mathrm{p}}$.

Os dados experimentais de do/dt foram selecionados de forma que apresentassem medidas em inter- valos de -t mais extensos possiveis. Nossa preocupação com isto foi a utilização de dados que exibissem as principais características da regiāo de difraçāo tais como: ponto ótico (do/dt) $t_{t=0}$ pico de dífraçāo, $1^{\circ}$. minimo ("dip"), $2^{\circ}$. máximo ("bump") e a região de queda após o $2^{\circ}$. máximo. Duas particularidades foram observadas e vale a pena tecer alguns comentários sobre elas. Primeira, a reaçāo $\pi \mathrm{p}$ a $50 \mathrm{GeV} / \mathrm{c}$ e embora tenha dados até $-t \sim 8(\mathrm{GeV} / \mathrm{c})^{2}$, não apresenta uma estrutura do tipo "dipbump", mas mostra apenas uma quebra ("break") biusca de d $\sigma / d t$ entre 3 e $4(\mathrm{GeV} / \mathrm{c})^{2}$, seguida de uma região com queda muito suave ("shoulder"). Segunda, os dados de $\pi^{*}$ p a $200 \mathrm{GeV} / \mathrm{c}$ se estendem até $-\mathrm{t}-3.5$ $(\mathrm{GeV} / \mathrm{c})^{2}$ e por isto não exibern o "dip", que nas reaçōes pion-próton é normalmente formado entre 3.8 e 4.0 $(\mathrm{GeV} / \mathrm{c})^{2}$. Uma vez que a existência da estrutura "dipbump" (ou mesmo "break-shoulder") é fundamental para uma análise completa e fisicamente correta da eiconal e tarnbem, que o "dip" já se formou em $\pi$ "p a $50 \mathrm{GeV} / \mathrm{c}$ (consequentemente deverá existir para energias maiores), fizemos a seguinte extrapolação para esta reação a $200 \mathrm{GeV} / \mathrm{c}$ : existe um "dip" entre $3.8-4.0(\mathrm{GeV} / \mathrm{c})^{2} \mathrm{e}$ que, após o mesmo, os dados de do/dt apresentam uma inclinaçäo semelhante a da mesma reação a 50 $\mathrm{GeV} / \mathrm{c}$.

As tabelas de dados de do/dt $x-t$ existentes nos artigos que divulgam os resultados de experiencias, geralmente não vem com o valor relativo ao ponto ótico. Assim, determinamos os pontos óticos através da seguinte expressão baseada no Teorema Ótico

$$
\left.\frac{\mathrm{d \sigma}}{\mathrm{dt}}\right\rangle_{-\mathrm{t}=0}\left[\mathrm{mb} /(\mathrm{GeV} / \mathrm{c})^{2}\right]=0.051\left(1+\rho^{2}\right) \sigma_{t o t}^{2}[\mathrm{mb}]^{2}
$$

Em nenhuma reaçáo elástica hádron-hádron a altas energias existem dados experimentais de dc/dt obtidos numa mesma experiência para tocia regiāo de difraçäo. Geralmente se fazem medidas na regiäo do pico de difração e na região de "dip-bump" separadamente. Assim, para cada energia há necessidade de se juntar dados de intervalos de -t diferentes. Fizemos essa união de dados tomando o cuidado de escolher somente aqueles normalizados e que se superpunham suavemente sem descontinuidade.

As tabelas 1 e 2 apresentam todas as informaçöes sobre as grandezas d $\sigma / d t, \sigma_{\text {tot }}$ e $\rho$ e, também sobre os pontos óticos calculados para as reaçōes $\pi p$ e $\pi^{+} p$ nas energias de $50 \mathrm{GeV} / \mathrm{c}$ e $200 \mathrm{GeV} / \mathrm{c}$ (AKERLOF et $\mathrm{al}^{1}$; ASA'D et $\mathrm{al}^{6}$; AYRES et $\mathrm{al}^{7}$; FAJARDO et $\mathrm{al}^{17}$. LIPKIN et $a^{24}$; RUBINSTEIN et $a^{28}$ ). 
TABELA 1 - informaçōes gerais sobre os dados experimentais de (do/dt)

\begin{tabular}{|c|c|c|c|c|c|c|}
\hline REAÇÄO & $\begin{array}{l}P_{\text {lat }} \\
(G e V / c)\end{array}$ & $\begin{array}{l}\mathrm{s}^{\tau / 2} \\
(\mathrm{GeV})\end{array}$ & REFERENCLAS & ACELERADOR & $\begin{array}{l}\text { INTERVALO } \\
\text { DE -t }(\mathrm{GeV} / \mathrm{c})^{2}\end{array}$ & $\begin{array}{l}\text { NÚMERO TOTAL } \\
\text { DE DADOS (m) }\end{array}$ \\
\hline$\pi^{*} p$ & 50 & 9.78 & $\begin{array}{l}\text { Ayres et al 1977) } \\
\text { Asa'd et al }(1985)^{\epsilon}\end{array}$ & $\begin{array}{l}\text { FERMILAB } \\
\text { CERN }\end{array}$ & $\begin{array}{l}0.0375 \leq-t \leq 0.8 \\
0.75 \leq-t \leq 7.5\end{array}$ & 44 \\
\hline$\pi^{+} p$ & 200 & 19.42 & $\begin{array}{l}\text { Akerlof ef al ( } 1976)^{1} \\
\text { Rubinstein et al }(1984)^{28}\end{array}$ & $\begin{array}{l}\text { FERMILAB } \\
\text { FERMILAB }\end{array}$ & $\begin{array}{l}0.075 \leq-\mathrm{t} \leq 1.75 \\
0.95 \leq-\mathrm{t} \leq 3.35\end{array}$ & 74 \\
\hline$\pi p$ & 50 & 9.78 & $\begin{array}{l}\text { Ayres et al }(1985)^{7} \\
\text { Asa'd et al }(1985)^{6}\end{array}$ & $\begin{array}{l}\text { FERMILAB } \\
\text { CERN }\end{array}$ & $\begin{array}{l}0.0375 \leq-\mathrm{t} \leq 0.75 \\
0.725 \leq-\mathrm{t} \leq 7.75\end{array}$ & 61 \\
\hline$\pi p$ & 200 & 19.42 & $\begin{array}{l}\text { Akerlof et al (1976) } \\
\text { Rubinstein et a! }(1984)^{2}\end{array}$ & $\begin{array}{l}\text { FERMILAB } \\
28 \text { FERMILAB }\end{array}$ & $\begin{array}{l}0.075 \leq-\mathrm{t} \leq 2.375 \\
0.95 \leq-\leq 10.25\end{array}$ & 81 \\
\hline
\end{tabular}

TABELA 2 - Dados experimentais de $\sigma_{\text {tot }}, p$ e $(\dot{d} \sigma / d)_{t=0}$ para as reaçōes $\pi^{+} p$ e $\pi^{p} p$.

\begin{tabular}{|c|c|c|c|c|c|}
\hline REAÇŌES & $\begin{array}{l}p_{1 \mathrm{ab}} \\
(\mathrm{GeV} / \mathrm{c})\end{array}$ & $\begin{array}{l}\mathrm{s}^{1 / 2} \\
(\mathrm{GeV})\end{array}$ & $\sigma_{\text {tot }}(m b)$ & $\rho=\operatorname{Ref}(s, 0) / \operatorname{Imf}(s, 0)$ & $(\mathrm{d} \sigma / \mathrm{dt})_{\mathrm{t}=0}\left[\mathrm{mb} /(\mathrm{GeV} / \mathrm{c})^{2}\right]$ \\
\hline$\pi^{*} p$ & 50 & 9.78 & $23.10 \pm 0.18$ & -0.06 (estimado) & $27.19 \pm 0.42$ \\
\hline$\pi^{+} p$ & 200 & 19.42 & $23.84 \pm 0.06$ & $0.053 \pm 0.017$ & $28.96 \pm 0.14$ \\
\hline$\pi p$ & 50 & 9.78 & $24.13 \pm 0.28$ & $-0.003 \pm 0.013$ & $29.45 \pm 0.29$ \\
\hline$\pi p$ & 200 & 19.42 & $24.33 \pm 0.04$ & $0.064 \pm 0.020$ & $30.27 \pm 0.10$ \\
\hline
\end{tabular}

A parametrização dos dados de seção de choque diferencial elástica foi feita mediante as seguintes condições:

(a) amplitude de espalhamento elástico é fundamentalmente imaginária e dada por

$$
a(s, t)=\sum_{i=s}^{n} \alpha_{i} e^{-\beta i(-t)} ;
$$

(b) esta amplitude tem um zero entre $-\mathrm{t}=3$ e $4(\mathrm{GeV} / \mathrm{c})^{2}$ (antes do zero ela é positiva, após o que troca de sinal);

(c) a seção de choque diferencial elástica é dada por

$$
\left.\frac{d \sigma}{d t}\right)_{\text {ajusto }}=\pi\left[\sum_{i=1}^{n} \alpha e^{-\beta i(-t)}\right]^{2}
$$

(d) o ajuste foi realizado através do método computacional dos mínimos quadrados para funções arbitrárias denominado GRIDLS (BEVINGTON ${ }^{9}$ - capí- tulo 11), onde os parâmetros $\alpha_{i}$ e $\beta_{i}$ sao obtidos pela minimizaçāo do $\chi^{2}$ dado por

$$
\chi^{2}=\sum_{i=1}^{m}\left[\frac{Y_{1}-Y\left(x_{i}\right)}{\sigma_{1}}\right]^{2},
$$

em que: $m=$ nümero de pontos experimentais, $\sigma_{i}=$ incerteza dos dados $Y_{i} \in Y\left(X_{i}\right)=$ valor da funçáo de ajuste na abcissa $x_{i}$, correspondente a $Y$; e finalmente,

e) os pontos da regiāo do "dip" $\left[3.0<-\mathrm{t}<4.5(\mathrm{GeV} / \mathrm{c})^{2}\right]$, onde a seção de choque diferencial elástica é dominada pela parte real da amplitude de espalhamento, foram retirados no processo de minimizaçăo $\chi^{2}$.

A tabela 3 apresenta os parâmetros de ajuste que encontramos para as reaçōes $\pi^{+} p$ e $\pi p$ nas energias de $50 \mathrm{GeV} / \mathrm{c}$ e $200 \mathrm{GeV} / \mathrm{c}$. A última linha desta tabela dá o valor de $\chi^{2}$ por graus de liberdade $(\mathrm{gdl})$. [gdl = número de pontos experimentais (m) menos o número de parâmetros de ajuste $(2 n)]$. 
TABELA 3 - Parâmetros de ajuste de (do/dt)rop calculados através da Eq. 12.

\begin{tabular}{|c|c|c|c|c|}
\hline REAÇĀO & $\pi^{+} \mathbf{p}$ & $\pi^{*} \mathbf{p}$ & $\pi p p$ & $\pi p$ \\
\hline$p_{\text {lab }}(\mathrm{GeV} / \mathrm{c})$ & 50 & 200 & 50 & 200 \\
\hline$s^{12}$ & $\quad 9.78$ & 19.42 & 9.78 & 19.42 \\
\hline$\alpha$ & $4.191 \pm 0.013$ & $4.488 \pm 0.161$ & $4.378 \pm 0.092$ & $4.544 \pm 0.012$ \\
\hline$\alpha_{z}$ & $1.797 \pm 0.005$ & $1.653 \pm 0.022$ & $1.801 \pm 0.028$ & $1.698 \pm 0.005$ \\
\hline$\alpha_{3}$ & $1.269 \pm 0.005$ & $-1.270 \pm 0.020$ & $-1.269 \pm 0.026$ & $-1.264 \pm 0.004$ \\
\hline$\alpha_{4}$ & $-0.0046 \pm 0.0003$ & $-.0049 \pm 0.0012$ & $-0.0030 \pm 0.0003$ & $-0.0040 \pm 0.0002$ \\
\hline$B_{1}$ & $4.384 \pm 0.015$ & $4.670 \pm 0.134$ & $4.579 \pm 0.088$ & $4.837 \pm 0.016$ \\
\hline$\beta_{2}$ & $1.521 \pm 0.002$ & $1.519 \pm 0.008$ & $1.532 \pm 0.012$ & $1.514 \pm 0.002$ \\
\hline$R_{3}$ & $1.470 \pm 0.002$ & $1.469 \pm 0.009$ & $1.464 \pm 0.015$ & $1.465 \pm 0.002$ \\
\hline $6_{4}$ & $0.363 \pm 0.015$ & $0.386 \pm 0.048$ & $0.365 \pm 0.026$ & $0.420 \pm 0.016$ \\
\hline$\chi^{2 / g d l}$ & $44 / 36$ & $117 / 66$ & $87 / 53$ & $142 / 79$ \\
\hline
\end{tabular}

Para uma descriçāo completa dos dados experimentais de (do/dt), è necessária a inclusão da parte real da amplitude de espalhamento. Utilizando-se as relações de dispersão (MARTIN ${ }^{25}$ ), podemos obter a seguinte expressão aproximada para Ref(s,t) a partir da Eq. 11

$$
\left.\operatorname{Ref}(s, t)=\rho \sum_{i=1}^{n} \alpha_{i}[\}-\beta_{i}(-t)\right] e^{-\beta i(-t)}
$$

Assim, nossa proposta de parametrizaçåo para a representação completa dos dados experimentais de do/dt na regiâo de diffaçăo é dada por

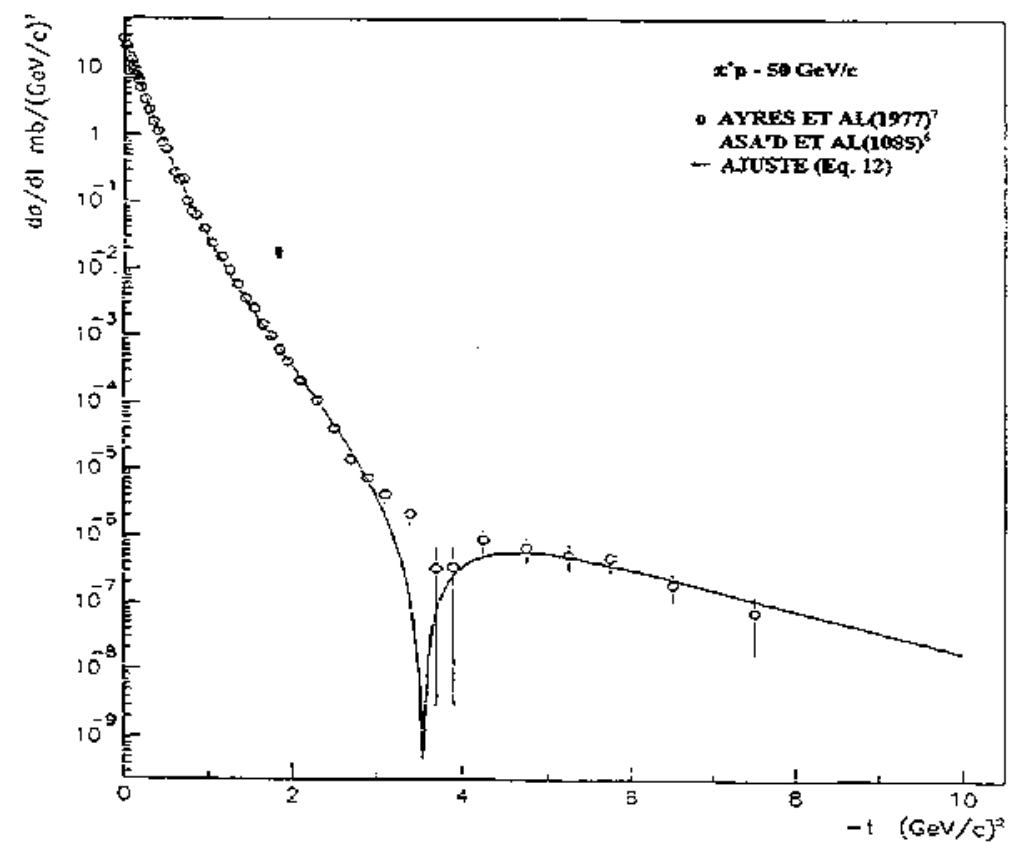

Figura 1 - Dados experimentais de seçāo de choque diferencial elástica de $\pi^{*} p$ a $p_{L b}=50 \mathrm{GeV} / c$ e curya de ajuste pela $E q-12$

$$
\begin{aligned}
& \frac{d \sigma}{d t}=\pi\left[\sum_{i=1}^{n} \alpha_{i} e^{-\beta i(-t)}\right]^{2}+ \\
& \pi \rho^{2}\left[\sum_{i=1}^{n} \alpha_{i}\left(1-\beta_{i}(-t)\right) e^{-\beta_{i}(-1)}\right]^{2}
\end{aligned}
$$

Podemos observar que para $\rho=0$ (ou seja, Ref(s,t) $=0$, a Eq. 15 se reduz à Eq. 12 de ajuste.

As figuras $1,3,5$ e 7 mostram os resultados dos ajustes (Eq. 12 ou Eq. $15 \mathrm{com} \rho=0$ ) em comparaçăo com os dados experimentais de $\pi^{+} p$ $50 \mathrm{GeV} / \mathrm{c}(\rho=-0.06), \pi^{+} p-200 \mathrm{GeV} / \mathrm{c}(p=0.053)$, $\pi \mathrm{p}-$ $50 \mathrm{GeV} / \mathrm{c}(\rho=-0.016)$ e $\pi p-200 \mathrm{GeV} / \mathrm{C}(\rho=-0.064)$ respectivamente.

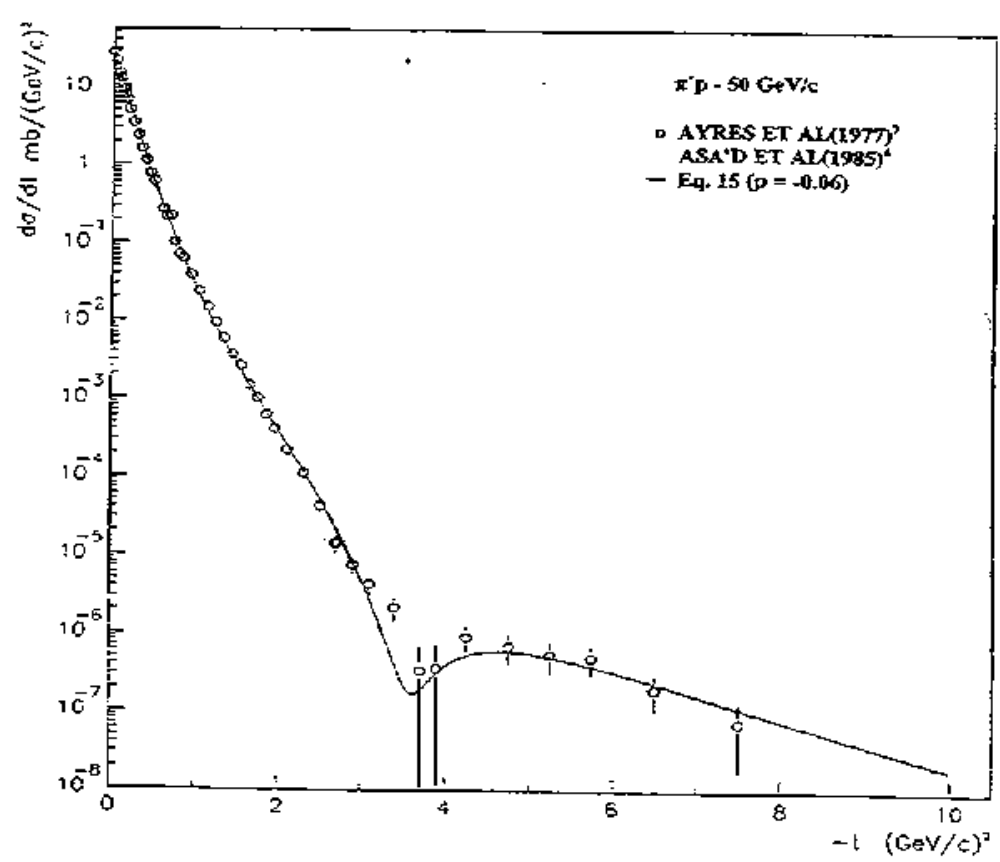

Figura 2 - Dados experimentais de secão de choque diferencial elástica de $\pi^{*} p$ a $p_{3 a p}=50 \mathrm{GeV} / \mathrm{c}$ e curva calculada através da Eq. $15 \operatorname{com} p=-0.06$ 


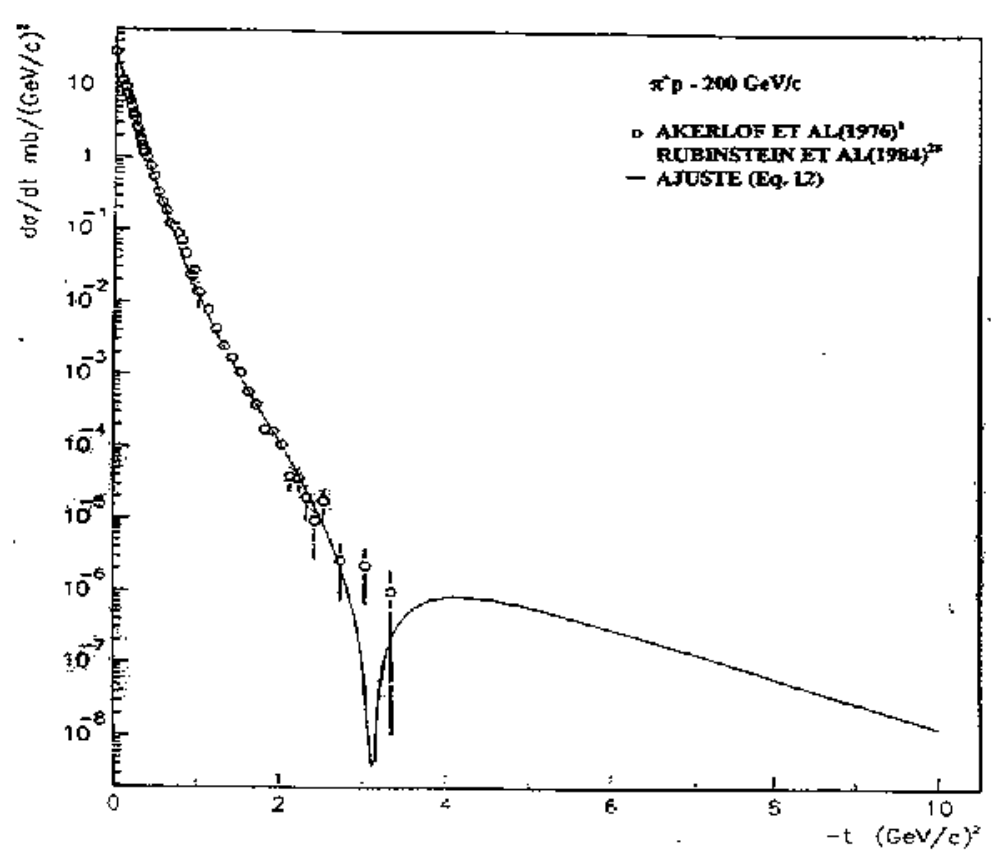

Figura 3 - Dados experimentais de seção de choque diferencial elástica de $\pi^{*} \mathrm{p}$ a $P_{\mathrm{lab}}=200 \mathrm{GeV} / \mathrm{c}$ e curva de ajuste pela Eq. 12

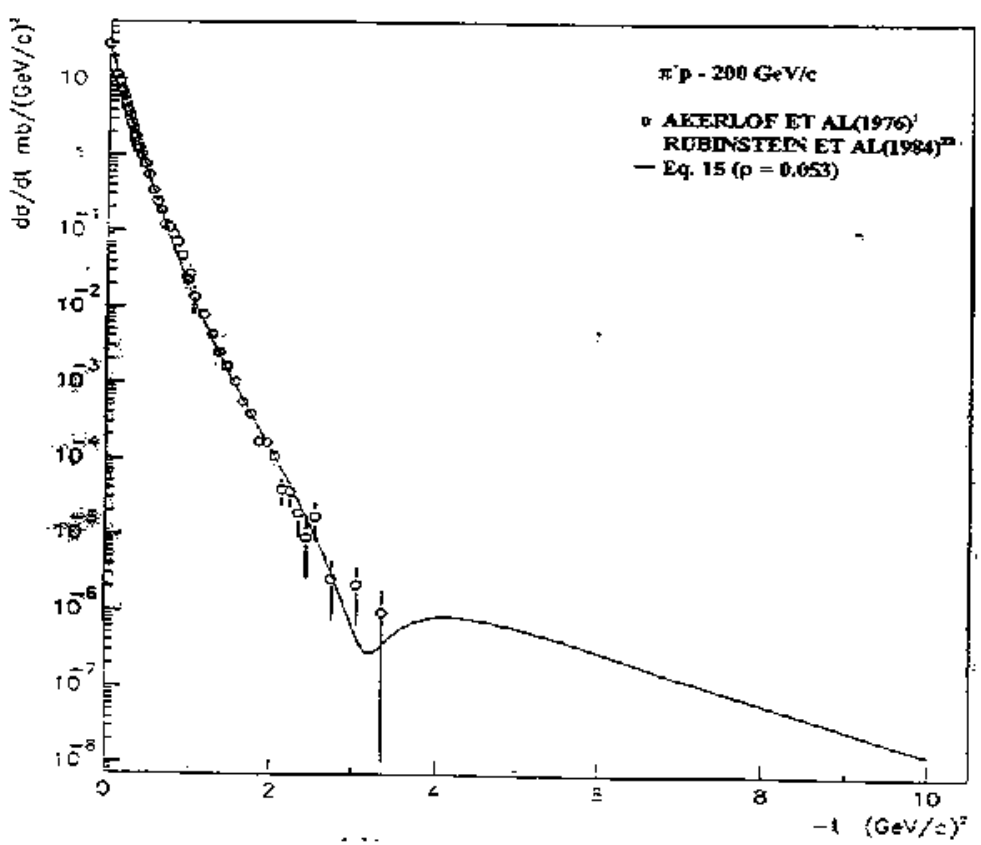

Figura 4 - Dados experimentais de seção de choque diferencial elástica de $\pi^{+} \mathrm{p}$ a $p_{\text {. }}=200 \mathrm{GeV} / \mathrm{c}$ e curva calculada através da Eq. $15 \mathrm{com} p=0.053$

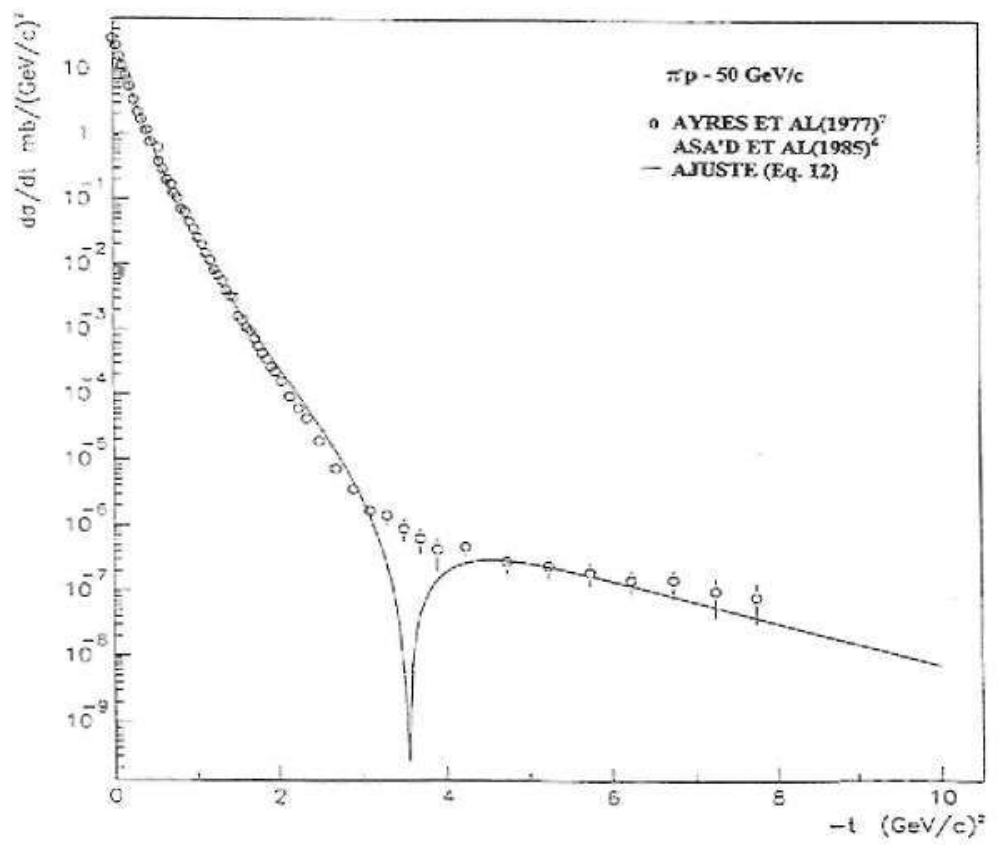

Figura 5 - Dados experimentais de seçäo de choque diferencial elástica de $\pi \mathrm{p}$ a $\mathrm{p}_{\mathrm{lab}}=50 \mathrm{GeV} / \mathrm{c}$ e curva de ajuste pela Eq. 12

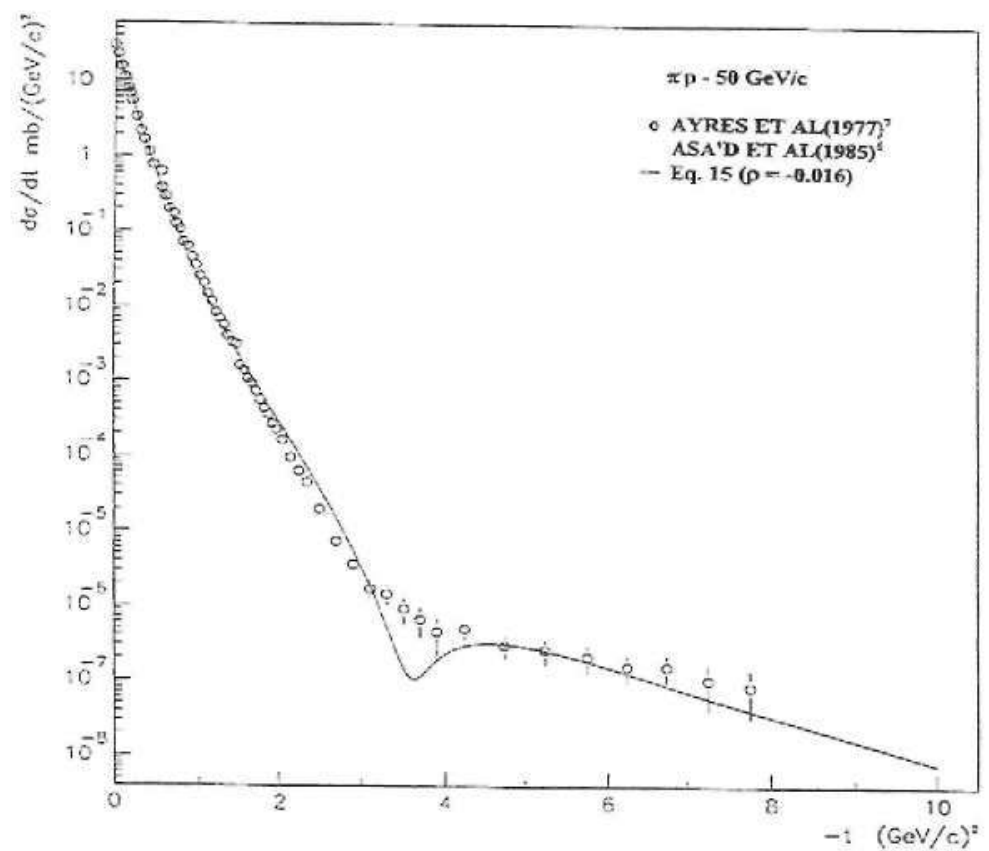

Figura 6 - Dados experimentais de seção de choque diferencial elástica de $\pi \mathrm{p}$ a $p_{\mathrm{tab}}=50 \mathrm{GeV} / \mathrm{c}$ e curva calculada através da Eq. $15 \operatorname{com} p=-0.016$ 


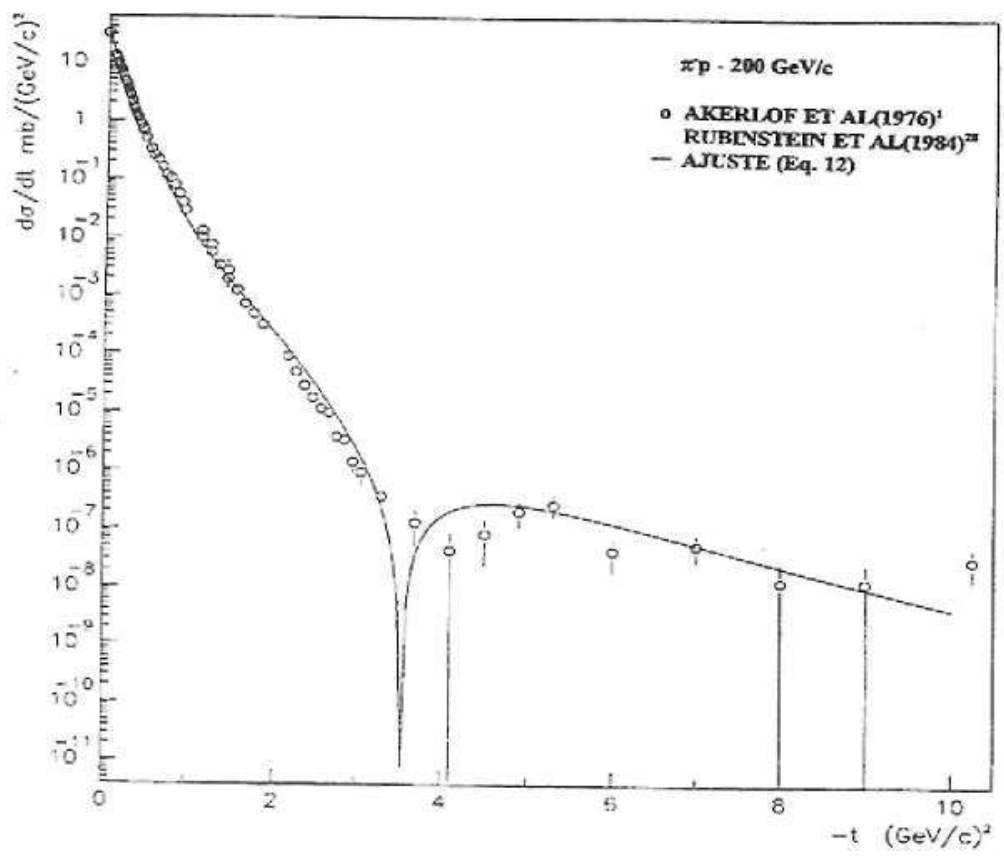

Figura 7 - Dados experimentais de seção de choque diferencial elástica de $\pi p$ a $p_{1 \mathrm{ab}}=200$ $\mathrm{GeV} / \mathrm{c}$ e curva de ajuste pela Eq. 12
De modo geral, nossa proposta de parametrização resultou numa boa descrição dos dados experimentais; apenas no caso de p p ocorreram alguns problemas, que no entanto, não são significativos na análise da eiconal. Para esta reação a $\mathrm{p}_{\mathrm{lab}}=50 \mathrm{GeV} / \mathrm{c}$, mesmo considerando o máximo valor de $\mathrm{p}$ (valor da grandeza + erro experimental), não foi possível descrever com precisão a região de pontos em torno de $-\mathrm{t}=3.5(\mathrm{GeV} / \mathrm{c})^{2}$. Primeiramente, por que em p p o "dip" ainda não se formou a esta energia. Existe apenas uma mudança brusca de inclinação e, conseqüentemente, deve estar presente algum outro efeito físico que não pode ser descrito pela Eq. 15. Esta expressão obtida como base nos argumentos de Martin (MARTIN ${ }^{25}$ ) é aproximada, mesmo para energias mais altas e para outras reações. Em segundo lugar, o valor de $p$ é muito pequeno para que a parte real da amplitude de espalhamento possa preencher todo o vazio (Fig. 5) deixado pela parte imaginária. Acreditamos que necessitamos generalizar o "gemetrical scaling" existente no segundo termo do segundo membro da Eq. 15. A p pab $=200 \mathrm{GeV} / \mathrm{c}$ a situação é um pouco pior. O ajuste não descreveu bem a região em -t - $2.5(\mathrm{GeV} / \mathrm{c})^{2}$ e encontrou um "dip" um pouco antes daquele simulado pelos dados. Aqui, além da limitação da Eq. 15, acreditamos que o que mais contribuiu para gerar esses problemas foi o comportamento dos dados experimentais acima de $-\mathrm{t}-3.5(\mathrm{GeV} / \mathrm{c})^{2}$. Existem dados com grandes erros, alguns com até $100 \%$ de incerteza e com uma distribuição de pontos bastante irregular.

Vale a pena ressaltar que os dados experimentais de ds/dt para as reações méson-próton são bem mais difíceis de se ajustarem através da Eq. 12 que os dados das reações núcleon-próton. Entendemos que os principais motivos para isto são: (1) as reações núcleonpróton (particularmente $\mathrm{pp}$ e $\mathrm{pp}$ ) apresentam medidas de $\mathrm{ds} / \mathrm{dt}$ mais completas, precisas, com distribuições de pontos mais regulares e a energias mais altas que as reações méson-próton; (2) do ponto ótico até o "dip", a seção de choque diferencial elástica de méson-próton, apresenta também um comportamento mais sinuoso que esta e, (3) em função do méson ser menor que o núcleon, a seção de choque diferencial elástica de méson-próton exibe um "dip" bem mais a frente [em -t $4.0(\mathrm{GeV} / \mathrm{c})^{2}$ ] do que no caso de núcleon-próton [em -t $\left.1.5(\mathrm{GeV} / \mathrm{c})^{2}\right]$.

O motivo (1) implica em sérias dificuldades de cálculo numérico para minimizar $o \chi^{2}$ e encontrar um aiuste ideal. Os motivos (2) e (3) exigem qe a Eq. 12 tenha pelo menos mais uma exponencial para as reações mésori-próton ( $n=4$, ou seja, 8 parāmetros livres) do que as reaçōes núcleon-próton (para estas bastam apenas 3 exponenciais, ou seja, 6 parânetros livres). Assim, quanto mais parâmetros fivres existirem, maiores serão as díficuldades para determiná-los, econseqüentemente, traverá ummaior comprometimento da qualidade da parametrizaçáo.

\section{CÁLCULO DA EICONAL}

Substituindo-se (11) e (14) em (6) e (7) respectivamente e tomando-se a transformada de Fourier-Bessel inversa temos

e

$$
\begin{gathered}
\operatorname{sen} \chi_{R}(s, b) e^{-s(s, b)}=\rho \sum_{i=1}^{n} \frac{\alpha_{i}}{2 \beta_{i}}\left(\frac{b^{2}}{4 \beta_{1}}\right) e^{-\frac{b^{2}}{4 \beta_{i}}} \\
\cos x_{R}(s, b) e^{-s(s, b)}=1-\sum_{i=1}^{n} \frac{\alpha_{i}}{2 \beta_{1}} e^{-\frac{b^{2}}{4 \beta_{i}}}
\end{gathered}
$$

Destas duas expressōes podemos obter onde

$$
\Omega(s, b)=\Omega_{0}(s, b)-1 / 2 \ln \left[1+\operatorname{tg}^{2} \chi_{R}(s, b)\right],
$$

$$
\begin{gathered}
\Omega_{\mathrm{c}}(\mathrm{s}, \mathrm{b})=-\ln \left[1-\sum_{i=1}^{n} \frac{\alpha_{i}}{2 \beta_{i}} e^{-\frac{b^{2}}{4 \beta_{i}}}\right] \\
\operatorname{tg} \chi_{\mathrm{R}}(\mathrm{s}, \mathrm{b}) \approx \frac{\rho \sum_{i=1}^{n} \frac{\alpha_{i}}{2 \beta_{i}} \frac{b^{2}}{4 \beta_{i}} e^{-\frac{b^{2}}{4 \beta_{i}}}}{1-\sum_{i=1}^{n} \frac{\alpha_{i}}{2 \beta_{i}} e^{-\frac{b^{2}}{4 \beta_{i}}}}
\end{gathered}
$$


A análise das expressōes (18), (19) e (20), usando os valores de $p$ (Tabela 2) e dos parâmetros de ajuste $\alpha$ e $\beta_{i}$ (Tabela 3), revelou que: (1) A parte real da eiconal, $\chi_{R}(\mathrm{~s}, \mathrm{~b})$, é nula em $\mathrm{b}=0$, e cresce, atingindo um máximo de $\sim 0.02$ entre $b=0.5$ e $0.6 \mathrm{fm}$ e depois decresce $a$ valores da ordem de $5 \times 10^{-4} \mathrm{em} \mathrm{b}=2 \mathrm{fm}$; (2) No intervalo de 0 a $2 \mathrm{fm}$, em geral $\Omega(\mathrm{s}, \mathrm{b})>>\chi_{R}(\mathrm{~s}, \mathrm{~b})$. Em relação aos valores de $\Omega(\mathrm{s}, \mathrm{b})$, os valores de $\chi_{R}(\mathrm{~s}, \mathrm{~b})$ correspondem $0 \%$ em $b=0,-4 \%$ em $b-0.5 \mathrm{fm},-10 \%$ em $b-1.0 \mathrm{fm}$, $\sim 20 \%$ em b $\sim 1.5 \mathrm{fm} \mathrm{e}-35 \%$ em b $=2 \mathrm{fm} \mathrm{e}$, (3) o $2^{\circ}$. termo do $2^{\circ}$. membro da Eq. 18 é muito pequeno, só altera os valores de $\Omega_{0}(\mathrm{~s}, \mathrm{~b})$ a partir do $4^{\circ}$. algarismo significativo.

Assim, em função das observações (1), (2) e (3), e de que os raios do pion e do próton são da ordem de 0.5 $\mathrm{fm}$ e $1.0 \mathrm{fm}$ respectivamente (o que nos leva a acreditar

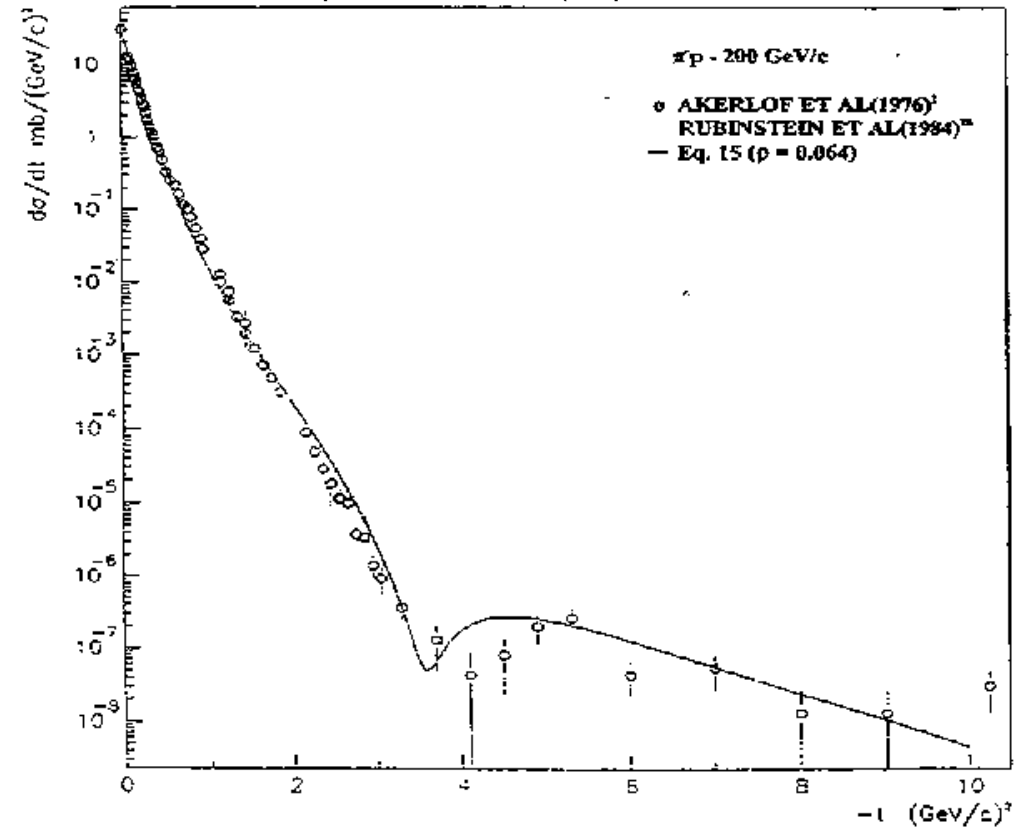

Figura 8 - Dados experimentais de seção de choque diferencial elástica de $\pi p$ a $p_{\text {lab }}=200$ GeV/c e a curva calculada através da Eq. $15 \mathrm{com} \rho=0.064$ que a maioria dos efeitos de interação forte entre eles ocorre até um b $-1.5 \mathrm{fm}$ ), é bastante razoável supor que o espalhamento pion-próton seja fundamentalmente difrativo onde o módulo da eiconal $\chi(s, b)$ é em boa aproximação, igual à opacidade $\Omega(s, b)$, onde esta é calculada através da Eq. 19.

Daqui para frente $\Omega(s, b)$ será chamada indistintamente de eiconal ou opacidade. A função $\chi_{k}(s, b)$ teria apenas alguma influência na regiāo do "dip".

As figuras 9 e 10 mostram os resultados da eiconal, calculada através da Eq. 19, em função do parāmetro de impacto, para as reaçöes $\pi^{+} p$ e $\pi p$ respectivamente. Em ambas as figuras, a linha continua corresponde a $p_{\text {lab }}=50 \mathrm{GeV} / \mathrm{c}$ e linha tracejada a $\mathrm{p}_{\mathrm{lab}}=$ $200 \mathrm{GeV} / \mathrm{c}$.

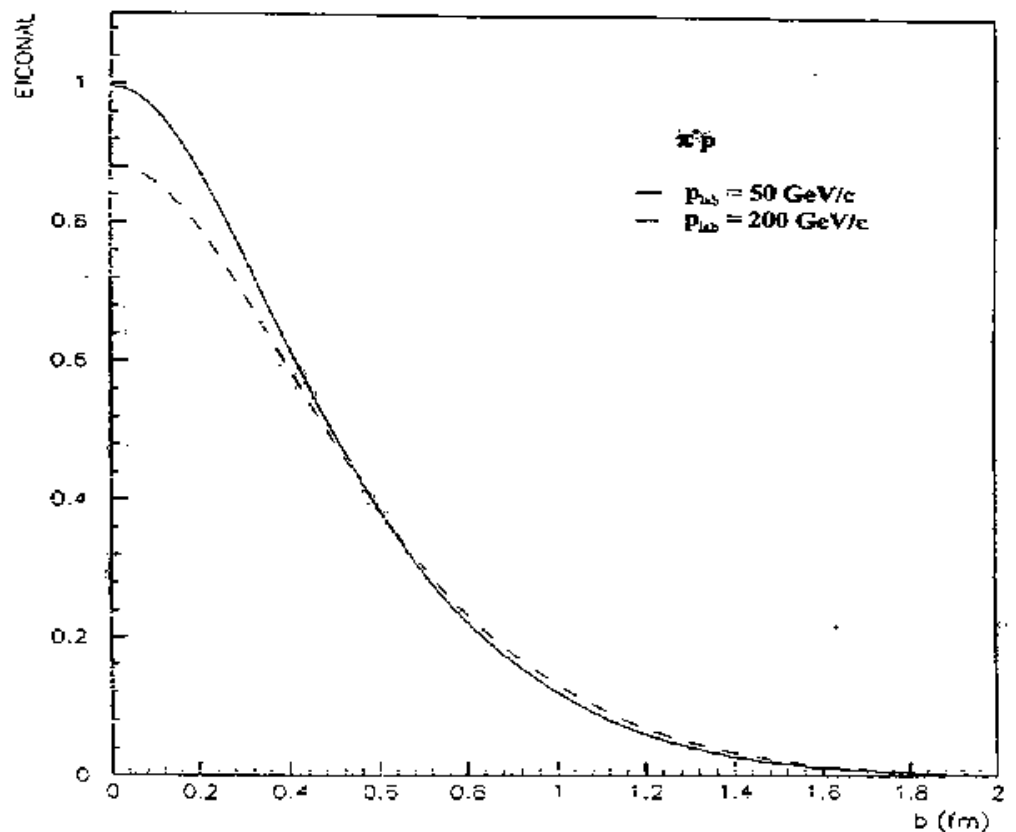

Figura 9 - Eiconal da reação $\pi^{+} p$ em função do parâmetro de impacto b. A curva continua corresponde a $p_{b 0}=\mathbf{5 0}$ GeV/c e a curva tracejada a $p_{1 a b}=200 \mathrm{GeV} / \mathrm{c}$

A tabela 4 mostra os mesmos resultados para alguns valores característicos de b.

TABELA 4 - Eiconal calculada através da Eq. 19 para alguns valores característicos do parâmetro de impacto b.

\begin{tabular}{llllllll}
\hline REA- & $P_{\mathrm{hb}}$ & $\mathrm{s}^{1 / 2}$ & $\Omega_{\mathrm{d}}(\mathrm{s}, \mathrm{b})$ & $\Omega_{\mathrm{o}}(\mathrm{s}, \mathrm{b})$ & $\Omega_{\mathrm{o}}(\mathrm{s}, \mathrm{b})$ & $\Omega_{\mathrm{o}}(\mathrm{s}, \mathrm{b})$ & $\Omega_{\mathrm{o}}(\mathrm{s}, \mathrm{b})$ \\
ÇĀO & & & $b=0 \mathrm{fm}$ & $b=0.5 \mathrm{fm}$ & $b=1.0 \mathrm{fm}$ & $b=1.5 \mathrm{fm}$ & $b=2.0 \mathrm{fm}$ \\
& $(\mathrm{GeV} / \mathrm{c})$ & $(\mathrm{GeV})$ & $b^{2}=0(\mathrm{GeV} / \mathrm{c})^{-2}$ & $b^{2}=6.42(\mathrm{GeV} / \mathrm{c})^{-2}$ & $b^{2}=25.68(\mathrm{GeV} / \mathrm{c})$ & $b^{2}=53.78(\mathrm{GeV} / \mathrm{c})$ & $b^{2}=102.73(\mathrm{GeV} / \mathrm{c})^{-2}$ \\
\hline
\end{tabular}

\begin{tabular}{lccccccc}
$\pi^{+} p$ & 50 & 9.78 & $0.996 \pm 0.009$ & $0.498 \pm 0.003$ & $0.121 \pm 0.001$ & $(0.179 \pm 0.002) \times 10^{-1}$ & $(0.137 \pm 0.002) \times 10^{-2}$ \\
$\pi \pi^{*} p$ & 200 & 19.42 & $0.882 \pm 0.058$ & $0.486 \pm 0.023$ & $0.132 \pm 0.001$ & $(0.221 \pm 0.016) \times 10^{-1}$ & $(0.197 \pm 0.026) \times 10^{-2}$ \\
$\pi p$ & 50 & 9.78 & $0.990 \pm 0.051$ & $0.508 \pm 0.016$ & $0.129 \pm 0.003$ & $(0.206 \pm 0.010) \times 10^{-1}$ & $(0.175 \pm 0.016) \times 10^{-2}$ \\
$\pi p$ & 200 & 19.42 & $0.902 \pm 0.008$ & $0.489 \pm 0.002$ & $0.136 \pm 0.001$ & $(0.240 \pm 0.002) \times 10^{-1}$ & $(0.233 \pm 0.003) \times 10^{-2}$ \\
\hline
\end{tabular}




\section{TESTES DA FATORAÇÃO E DO "GEOMETRICAL SCALING".}

Para a verificação das hipóteses da fatoração e do "geométrical scaling", vamos fazer um breve esclarecimento sobre estas questões.

O modelo eiconal de Chou-Yang (CHOU \& YANG ${ }^{14}$ ), na sua formulação original fixou a dependência em -t e supôs que, no regime de altas energias o espalhamento hadrônico elástico era puramente difrativo e independente da energia $\left[\mathrm{s}^{1 / 2}\right]$. Assim, a eicona! proposta pelo modelo foi da seguinte forma

$$
\Omega(b)=K D(b)
$$

onde $D$ (b) é a convoiução da densidade de matéria hadrônica e $\mathrm{K}$ é o coeficiente ou constante de absorção, uma constante característica de cada tipo de reação.

A não incorporação da dependência com a energia na eiconal, logo gerou problemas para o modelo de Chou-Yang, pois com a constatação experimentai, em 1973, de um crescimento da seção de choque total (AMALDI et $\mathrm{al}^{3}$; AMENDOLIA et $\mathrm{al}^{5}$ ) com o aumento da energia, para a reação pp na região do ISR - CERN, a "constante" de absorção e conseqüentemente a eiconai deveria de algum modo depender da energia.

A primeira tentativa de implementar esta idéia foi feita por Hayot e Sukhatme em 1974 (HAYOT \&
SUKHATME ${ }^{22}$ ), supondo-se o coeficiente de absorção variável com a energia. Assim, a eiconai foi escrita na forma fatorável

$$
\Omega(s, b)=K(s) D(b)
$$

Esta hipótese também apresentou problemas, pois como se sabe, ela não se verificou num cuidadoso estudo feito em 1980 para a reação pp na região do ISR CERN (AMALDI \& SCHUBERT ${ }^{4}$ ).

Se uma eiconai é fatorável segundo a expressão (22), a razão entre eiconaís para duas energias diferentes $s_{1}$ e $s_{2}$ independe da forma da distribuição da matéria na interação, ou seja, do parâmetro de impacto b. Assim, a razão

$$
\frac{\Omega\left(s_{1}, b\right)}{\Omega\left(s_{2}, b\right)}=\frac{K\left(s_{1}\right)}{K\left(s_{2}\right)}
$$

é uma constante com relação a b.

As figuras 11 e 12 mostram os resultados dos cálculos da razăo (23) para as reaçōes $\pi^{+} p$ e $\pi^{*}$ p respectivamente, tomando-se $\left(s_{1}\right)^{1 / 2}=19.42 \mathrm{GeV}$ (ou $p_{\mathrm{tab}}=200$ $\mathrm{GeV} / \mathrm{c}$ ) e $\left(\mathrm{s}_{2}\right)^{1 / 2}=9.78 \mathrm{GeV}$ (ou $p_{1 a b}=50 \mathrm{GeV} / \mathrm{c}$ ). A. figura 13 faz uma comparaçāo dos resultados das figuras 11 e 12. Para uma melhor visualização, mostramos, nesta figura, apenas os valores das razōes (na forma de linhas) sem as barras de erros.

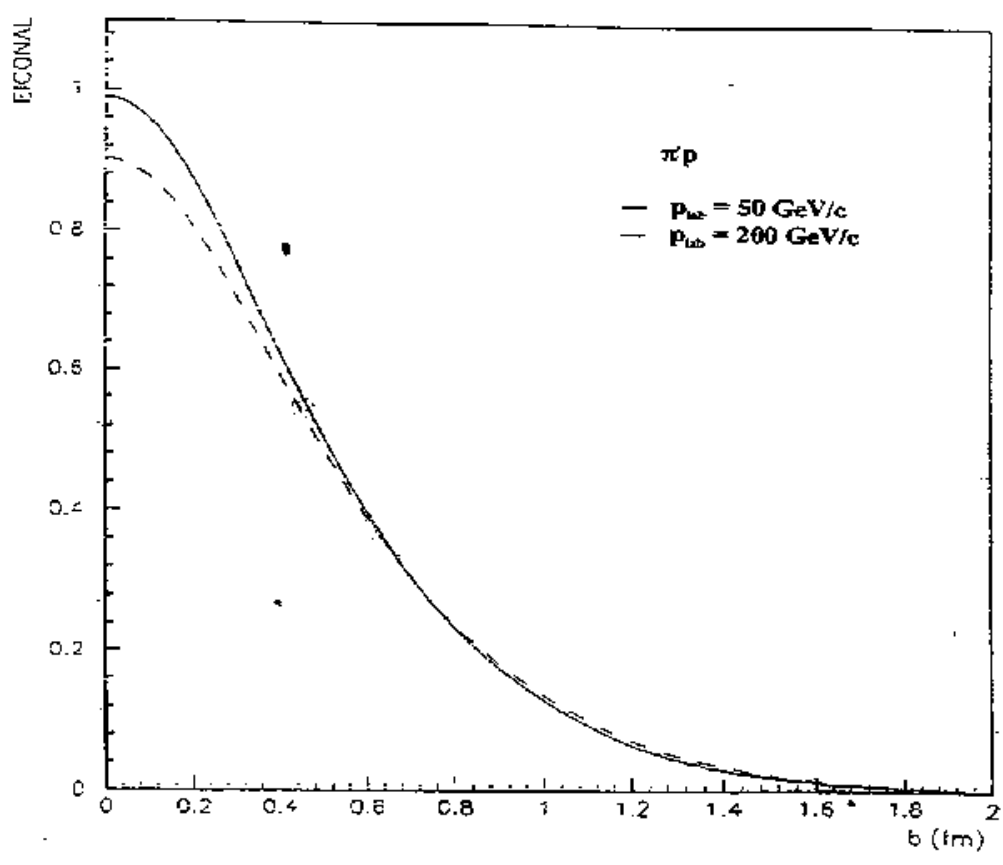

Figura 10 - Eiconal da reaçāo $\pi$ p em funçāo do parámetro de impacto $b$. A curva continua corresponde a $p_{\text {bb }}=\mathbf{5 0}$ GeV/c e a curva tracejada a $P_{\text {Lob }}=200 \mathrm{GeV} / \mathrm{c}$

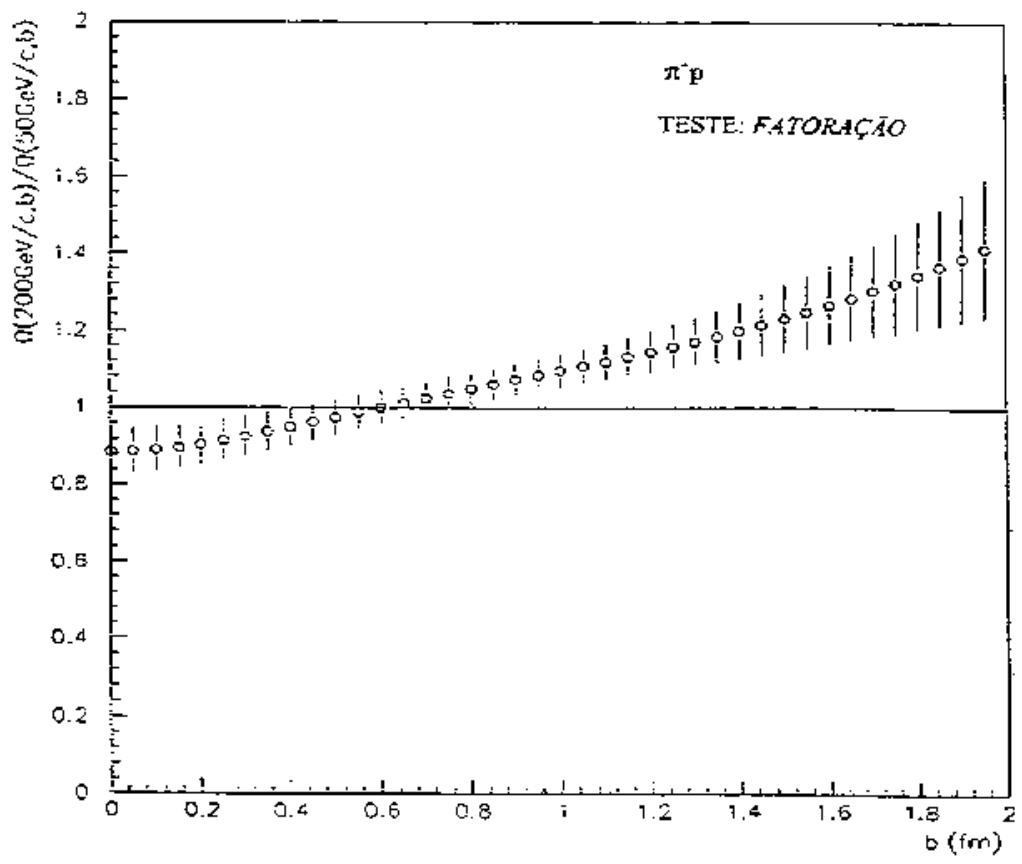

Figura 11 - Razäo entre as eiconais da reação $\pi^{*} p$ a $p_{\text {lat }}=$ $200 \mathrm{GeV} / \mathrm{c}$ e $p_{\mathrm{tat}}=50 \mathrm{GeV} / \mathrm{c}$ em funçăo do parâmetro de impacto b 


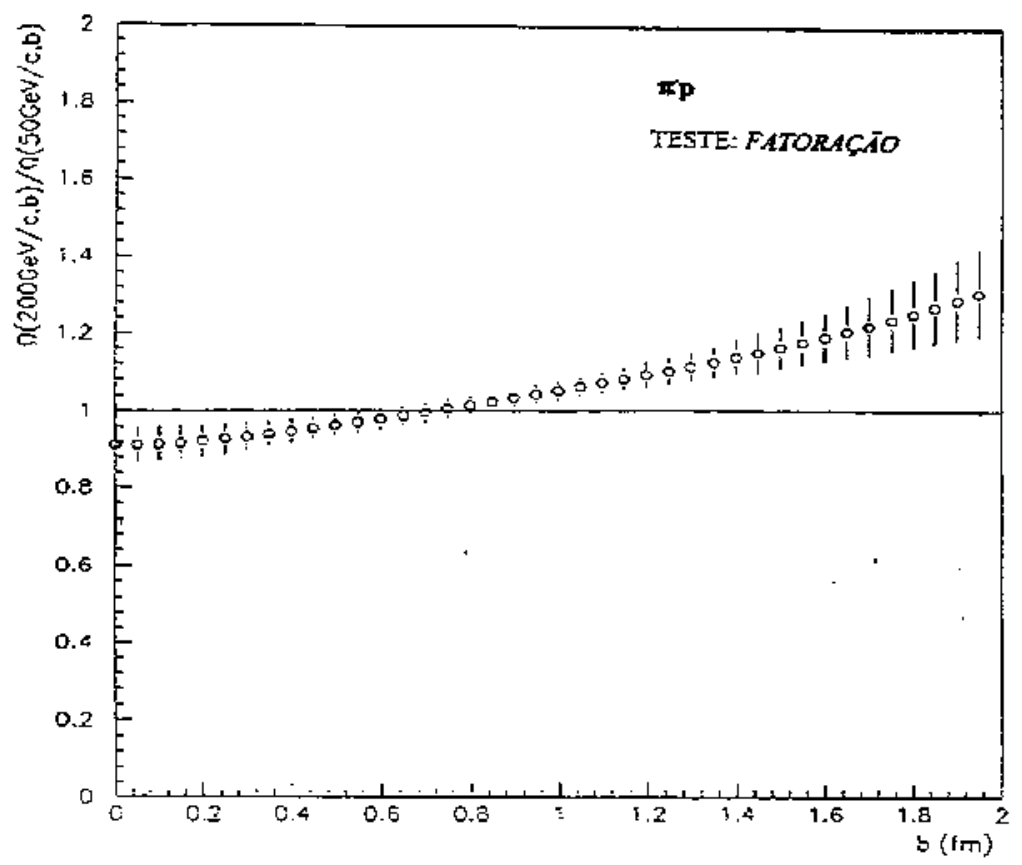

Figura 12 - Razào entre as eiconais da reação $x p$ a $p_{\text {lab }}=$ $200 \mathrm{GeV} / \mathrm{c}$ e $P_{\mathrm{bs}}=\mathbf{5 0} \mathrm{GeV} / \mathrm{c}$ em funçāo do parámetro de impacto b

Uma outra proposta alternativa para o comportamento da eiconal com relação à energia foi o "geometrical scaling", formulado por J. Dias de Deus em 1973 (DIAS DE DEUS ${ }^{16}$ ) e por A.J. Buras e J. Dias de Deus em 1974 (BURAS \& DiAS DE DEUS ${ }^{10}$ ).

O "geometrical scaling" estabelece essencialmente uma invariância de escala da distribuição transversal (parâmetro de impacto) da matéria hadrônica. De forma mais precisa afirma que numa colisão hádron-hádron a altas energias, a eiconal é uma função de uma única variável de escala $b^{*}$, ou seja,

$$
\begin{gathered}
\Omega(\mathrm{s}, \mathrm{b}) \rightarrow \Omega\left(\mathrm{b}^{*}\right) \\
\mathrm{s} \rightarrow \infty
\end{gathered}
$$

onde $b^{*}=b / R(s)$ e $R(s)$ é o raio de interação efetiva. Toda dependência energética está contida no raio de interação e, s e b não são variáveis independentes, mas estão correlacionadas.

$\mathrm{O}$ "geometrical scaling" perfeito entre duas eiconals para duas energias diferentes $s_{1}$ e $s_{3}$, deve ser satisfeita a seguinte condição (BELLANDI et $\mathrm{al}^{8}$; PÁDUA ${ }^{26}$ )

$$
\Omega\left(b, s_{1}\right)-\Omega\left(b / r, s_{2}\right)=0
$$

onde

$$
1 \leq r=\frac{R\left(s_{1}\right)}{R\left(s_{2}\right)} \leq \sqrt{\frac{\sigma_{t o t}\left(s_{1}\right)}{\sigma_{t o t}\left(s_{2}\right)}}
$$

A figura 14 apresenta o resultado do cálculo da Eq. 25 para a reaçäo $\pi^{*} p \operatorname{com}\left(s_{1}\right)^{1 / 2}=19.42 \mathrm{GeV}\left(\mathrm{p}_{\mathrm{Lab}}=\right.$

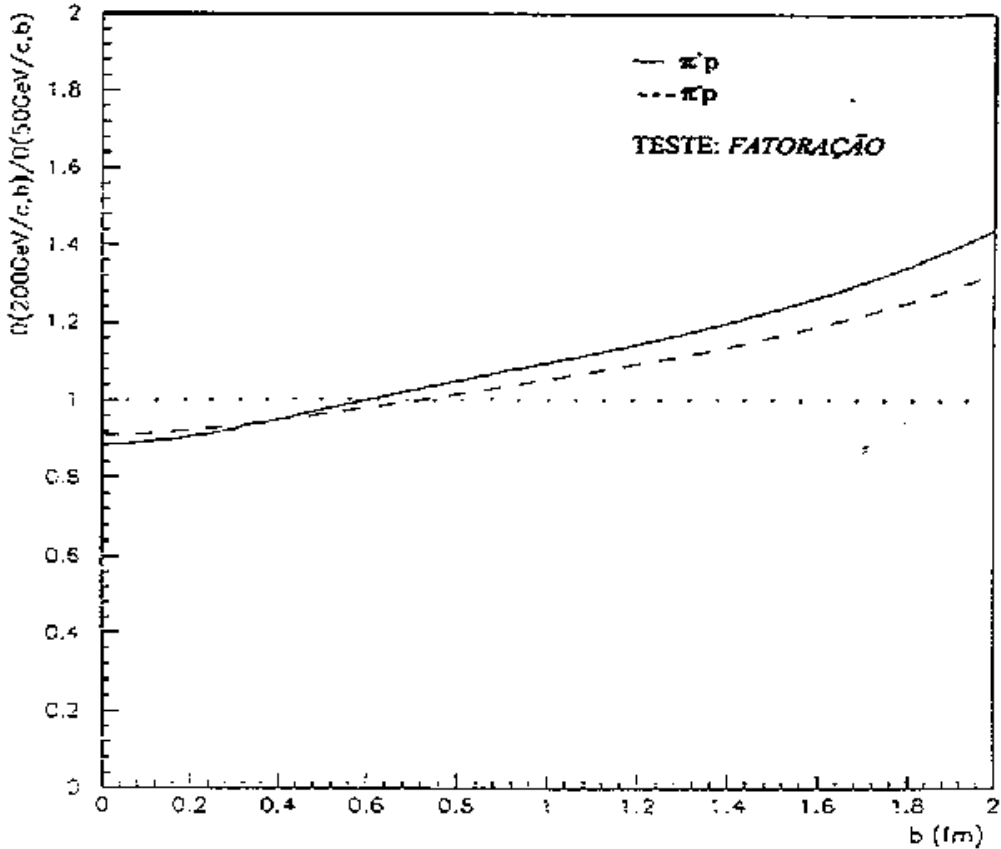

Figura 13 - Comparaçāo entre os resultados apresentados nas figuras 11 e 12

$200 \mathrm{GeV} / \mathrm{c})$ e $\left(\mathrm{s}_{2}\right)^{1 / 2}=9.78 \mathrm{GeV}\left(\mathrm{p}_{\text {lab }}=50 \mathrm{GeV} / \mathrm{c}\right)$ e com $r=1.016$. A figura 15 apresenta o mesmo para a reaçâo $\pi p$ apenas com $r=1.004$. Estes valores de $r$ foram calculados utilizando-se os dados de seção de choque total da Tab. 2 e tomando-se os máximos valores possíveis da Eq. 26. A figura 16 mostra uma comparação dos resultados das figuras 14 e 15. Para uma melhor visualização, mostramos, nesta figura, apenas os valores das razões (na forma de linhas) sem as barras de erros.

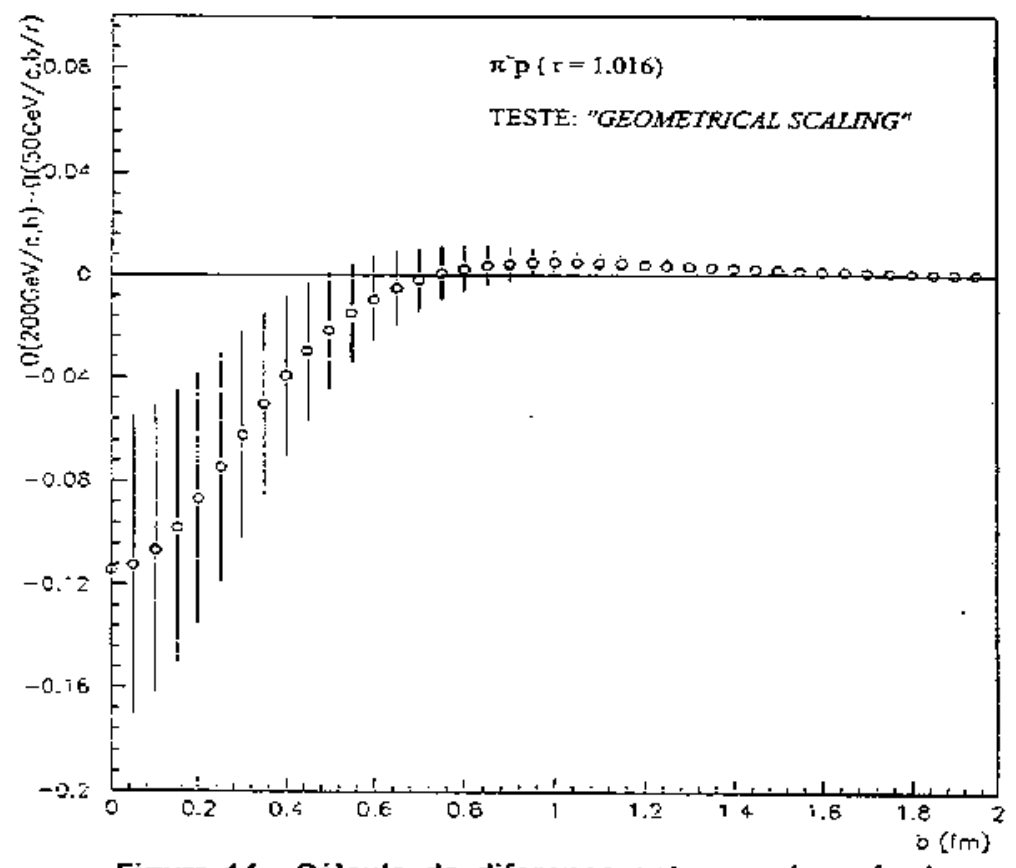

Figura 14 - Cálculo da diferença entre as eiconais da reaçäo $\pi^{*}$ p nas energias de $200 \mathrm{GeV} / \mathrm{c}$ e $50 \mathrm{GeV} / \mathrm{c}$ através da Eq. $25 \mathrm{com} r=1.016$ 


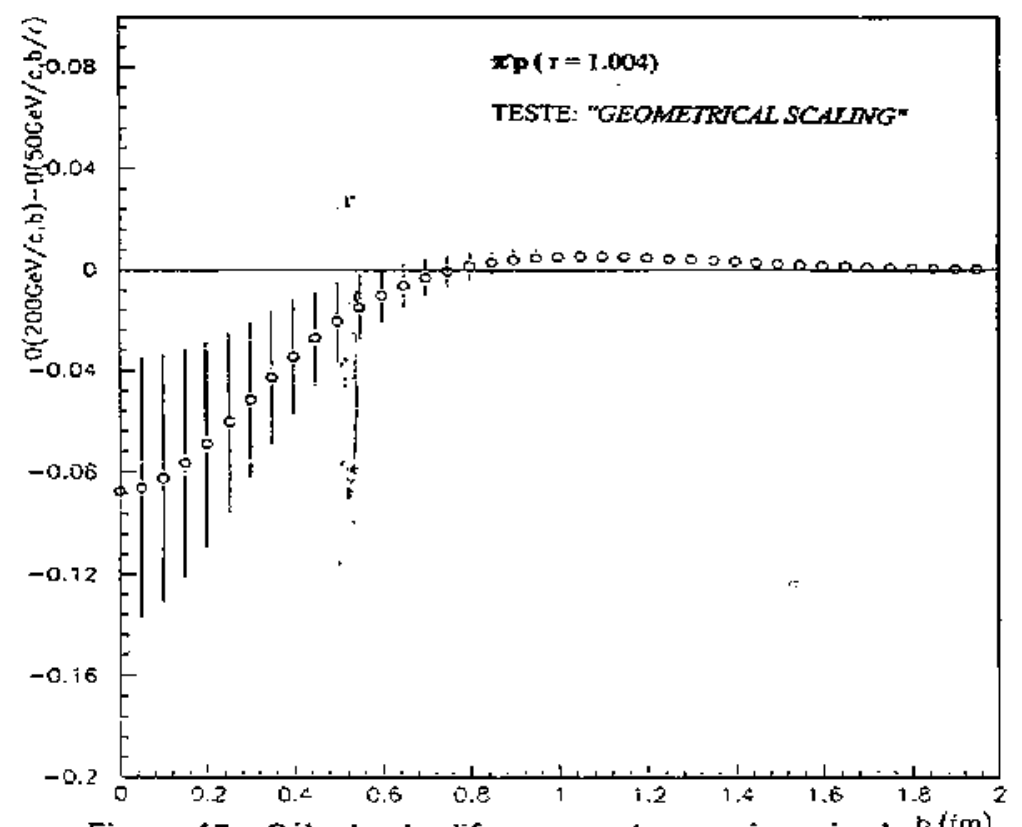

Figura 15 - Cálculo da diferença entre as eiconais da ${ }^{\circ}(\mathrm{im})$ reaçāo $\pi$ p nas energias de $200 \mathrm{GeV} / \mathrm{c}$ e $50 \mathrm{GeV} / \mathrm{c}$ através da Eq. $25 \mathrm{com} r=1.004$

\section{DISCUSSÃO DOS RESULTADOS E CONCLUSŌES}

As figuras 9 e 10 mostram claramente que para ambas as reaçōes as eiconais se cruzam em $b \sim 0.6$ fm para $\pi^{*} p$ e em $b-0.7$ fm para $\pi p$, quando caiculadas a $p_{\text {tab }}=50 \mathrm{GeV} / \mathrm{c}$ e $p_{\text {lab }}=200 \mathrm{GeV} / \mathrm{c}$. Para as duas reaçōes a eiconal a $p_{\text {lab }}=50 \mathrm{GeV} / \mathrm{c}$ é maior que a eiconal a $p_{\text {lab }}=200 \mathrm{GeV} / \mathrm{c}$ na regiāo central e menor na periférica. A maior diferença entre eiconais se verifica em $b=0$. Mesmo considerando os limites extremos dos erros em $\Omega$, esta diferença é significativa, o que é uma forte evidência da violação do "geometrical scaling" em $b=0$.

O fato de que a eiconal para os dois tipos de colisőes è maior para $\mathrm{p}_{\mathrm{lab}}=50 \mathrm{GeV} / \mathrm{c}$ na regiào central e menor na regiāo periférica do que para $p_{\text {lat }}=200 \mathrm{GeV} / \mathrm{c}$, nos leva a concluir que o aumento (embora muito pequeno) verificado em $\sigma_{\text {tot }}$ (Tabela 2) entre estas energias é conseqüência basicamente de fenômenos periféricos.

É USual definir transparência de uma interaçāo como $1-\mathrm{G}_{\text {in }}(\mathrm{b}=0)=\exp [-2 \Omega(\mathrm{b}=0)]$, o que corresponde à probabilidade de não ocorrer absorção em uma colisão frontai. A transparência para as colisōes pion-próton $\dot{e} \mathrm{de}-14 \%$ a $\mathrm{p}_{\mathrm{lat}}=50 \mathrm{GeV} / \mathrm{c}$ e de $\sim 17 \%$ a $\mathrm{p}_{\mathrm{lab}}=200$ $\mathrm{GeV} / \mathrm{c}$. Estes resultados mostram que as reações pionpróton são menos transparentes que as kaon-próton, uma vez que a transparência destas últimas está em torno de $20 \%$ (COVOLAN et al ${ }^{15}$ ).

Nossos resultados sobre fatoraçăo e "geometrical scaling" (figuras de 11 a 16) mostram claramente que as eiconais das reaçōes pion-próton não se fatorizam na regiäo periférica para $b>0.6 \mathrm{fm}$. Na regiăo central, entre 0 e $-0.6 \mathrm{fm}$, a razäo entre as eiconais a $p_{\mathrm{lab}}=200$

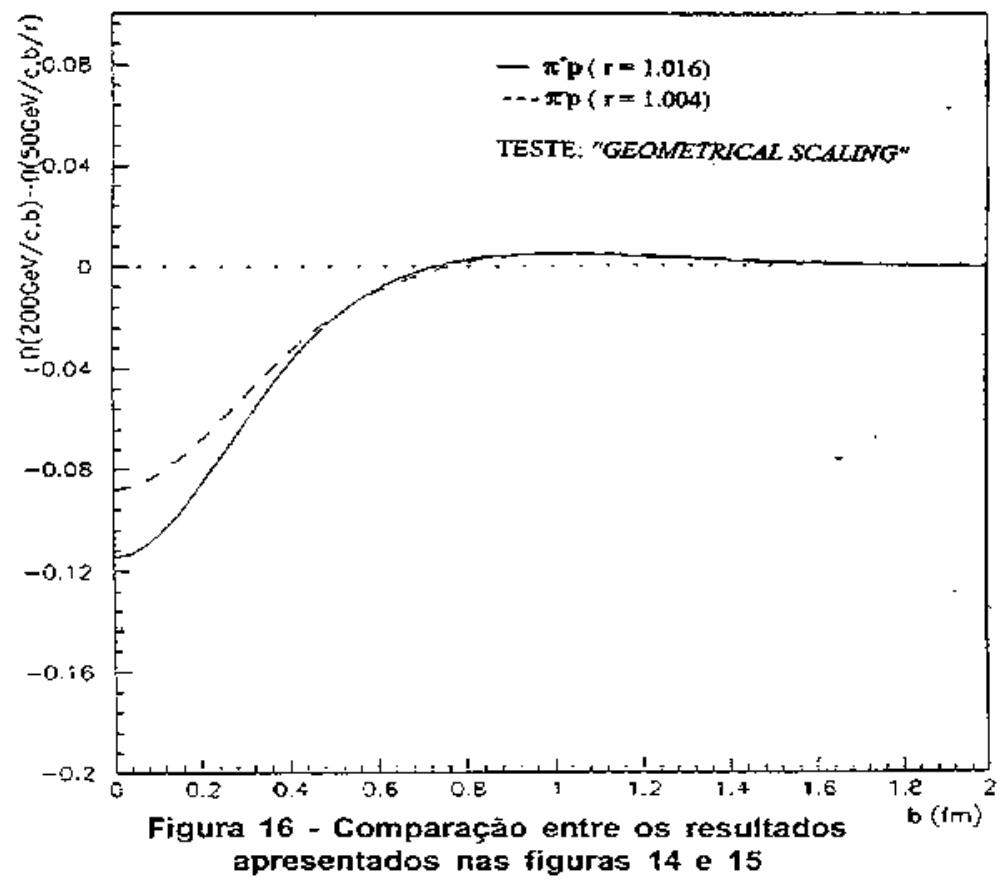

$\mathrm{GeV} / \mathrm{c}$ e $\mathrm{p}_{\mathrm{lab}}=50 \mathrm{GeV} / \mathrm{c}$ tem uma variaçào muito pequena, de tal forma que, se considerarmos os limites das barras de erros, ela poderá ser admitida como praticamente constante e com valor $\sim 0.94$ para $\pi^{+} p$ e -0.96 para $\pi$ p. No caso do "geometrical scaling" ocorre o contrário: ele se verifica com boa aproximaçāo para b > $0.6 \mathrm{fm}$, mas é violado na regiāo central.

Estes resultados concordam, pelo menos qualitativamente com os nossos obtidos para as reações pp e $\mathrm{p} \overline{\mathrm{p}}$ (PÁDUA ${ }^{26}$; BELLANDI et $\mathrm{al}^{6}$ ), mas discordam, por incrivel que possa parecer, com os de kaon-proton (COVOLAN et al15) realizado em faixas de energias equivalentes às do presente trabalho. No caso das reaçóes kaon-próton o "geometrical scaling" se verificou em toda regiāo de parametro de impacto entre 0 e $2 \mathrm{fm}$. Cabe aqui uma observaçāo que foi fundamental para este nosso estudo de pion-próton se tornasse mais confiável. $O$ comportamento da eiconal na região central é muito sensivel ao ajuste de do/dt na regiāo de grandes valores de -t, dai o motivo de escolhermos somente os dados experimentais que apresentassem a estrutura de "dipbump" (ou "break-shoulder") e realizarmos todos os ajustes (Eq. 12) com um zero em $-t \sim 4\left(\mathrm{GeV} / \mathrm{c}\right.$ ) ${ }^{2}$. Fizemos, a titulo de averiguação, os ajustes de $\pi^{+} \mathrm{p}$ a $\mathrm{p}_{\text {lab }}=200$ GeV/c e de $\pi$ p a $p_{\text {lab }}=50 \mathrm{GeV} / \mathrm{c}$ sem zeros na amplitude de espalhamento, e os resultados da eiconal se atteram significativamente, gerando até mesmo incoerências com relação ao comportamento dos dados experimentais de $\sigma_{\text {tot }}$ em função da energia para estas reações. Assim, acreditamos que existam problemas com os testes da fatoraçāo e do "geometrical scaling" na regiāo central para as reaçōes kaon-próton, uma vez que apenas K+p a $50 \mathrm{GeV} / \mathrm{C}$ apresenta estrutura de "dip- 
bump" e que isto, provoca um zero na amplitude de espalhamento. Para os demais dados de kaon-próton, $\mathrm{ds} / \mathrm{dt}$ foi medida no máximo até $-2(\mathrm{GeV} / \mathrm{c})^{2}$, ou seja, bem antes do "dip" que com certeza deve existir. Em nosso trabalho (COVOLAN et $\mathrm{al}^{15}$ ) este "dip" não foi levado em conta, e nem havia forma de fazê-lo, com o tipo de ajuste adotado (o mesmo da Eq. 12) e com os dados experimentais existentes.

Em resumo, nosso presente estudo mostra que a eiconal como função da energia para as reações pionpróton, deve ter duas componentes: uma fatorável, dominante na região central, e outra consistente com o "geometrical scaling" na região periférica.

Estes resultados de comportamento da eiconal estão coerentes com os dados experimentais de $\mathrm{S}_{\mathrm{el}} / \mathrm{S}_{\text {tot }}$ e de $\mathrm{S}_{\text {tot }}$ com relação à energia. O decréscimo da opacidade na região central para $\mathrm{p}^{+} \mathrm{p}$ e $\mathrm{p}-\mathrm{p}$ na transição de $50 \mathrm{GeV} / \mathrm{c}$ para $200 \mathrm{Ge} \mathrm{V/c} \mathrm{justifica} \mathrm{a} \mathrm{queda} \mathrm{apre-}$ sentada pelos dados experimentais de $\mathrm{S}_{\mathrm{el}} / \mathrm{S}_{\text {tot }}$ para estas reações, ou seja, neste aumento de energia, esta razão passa de $0.144 \pm 0.012$ para $0.130 \pm 0.012 \mathrm{em} \mathrm{p}^{+} \mathrm{p}$ e de $0.142+0.012$ para $0.129 \pm 0.012 \mathrm{em} \mathrm{p}$ p (AKERLOF et al $\left.{ }^{1}\right)$. No caso de Stot embora haja, segundo nossa análise, uma diminuição da eiconal na região central, o pequeno crescimento de $\mathrm{S}_{\text {tot }}$ verificado nesta transição de energia (veja dados na Tabela 2) está associado, principalmente, a uma expansão pura (sem variação da opacidade) da distribuição da matéria hadrônica na região periférica onde ocorre o "geometrical scaling".
Finalmente, gostaríamos de ressaltar que com este trabalho sobre as reações $\mathrm{p}^{+} \mathrm{p}$, completamos nossos estudos sobre a dependência energética na eiconal para espalhamentos hádron-hádron, feitos com base em ajustes numéricos dos experimentais $\mathrm{de} \mathrm{ds} / \mathrm{dt}$, ou seja, a partir de um método que independe de modelos teóricos.

Nossa conclusão geral sobre o assunto é a seguinte: "o problema da dependência energética da eiconal para as colisões hádron-hádron, embora não tenha mais incitado tanto interesse, ainda permanece uma questão em aberto, e nossos cálculos indicam que as hipóteses da fatoração e do "geometrical scaling" não são mutuamente excludentes como normalmente são tratadas, mas elas coexistem para uma mesma reação; apenas tem validades em regiões diferentes com relação ao parâmetro de impacto. Na região central a eiconal é aproximadamente fatorável e, na região periférica, seu comportamento é consistente com o "geometrical scaling". Assim, podemos escrevê-la como

$$
\Omega(s, b)=\Omega^{\text {central }}(s, b)+\Omega^{\text {periterica }}(s, b)
$$

onde $\Omega^{\text {central }}(\mathrm{s}, \mathrm{b})=\mathrm{K}(\mathrm{s}) \mathrm{D}(\mathrm{b})$ e $\Omega^{\text {pentenca }}(\mathrm{s}, \mathrm{b})=\Omega^{\prime}(\mathrm{b} / \mathrm{R}(\mathrm{s}))$. A expressão (27) é geral, válida para $p^{ \pm} p, \pi^{ \pm} p$ e $K{ }^{ \pm} p$, e para todas energias acima de $(s)^{1 / 2} \sim 10 \mathrm{GeV}\left(p_{\mathrm{l}=0}=50\right.$ $\mathrm{GeV} / \mathrm{c}$ ), mudam-se apenas as intervalos do parametro de impacto da validade de cada um de seus termos do segundo mernbro.

PÁDUA, A.B. de; DIAS DE DEUS, J. Analysis of the eikonal in pion-proton elastic collisions at high energies.

Semina: Ci. Exatas/Tecnoiógicas, Londrina, v. 16, n. 4, p. 516-528, Dec. 1995.

ABSTRACT: At the $p_{l a b}=50 \mathrm{GeV} / \mathrm{c}$ and $p_{l a b}=200 \mathrm{GeV} / \mathrm{c}$, on the range $\left.0<-t<10(\mathrm{GeV} / \mathrm{c})^{2}\right]$, we analysed the elastic scattering pion-proton on the impact parameter space in a model independent way. Firstly, we fitted the data by assuming a purely imaginary elastic amplitude and expressing it as a sum of the exponentials on the rnomentum transfer-t Secondly, we incorporated the real part of the amplitude by means of the dispersion relations. After that we calculated the eikonal and tested the geometrical scaling and factorizing eikonal models. Our results suggest an eikonal with a central factorízing part and a more peripherical part indicating a geometrical scaling.

KEY-WORDS: Elastic Scattering, High Energies, Pion, Proton, Elastic Differential Cross Section, Elastic Scattering Amplitude, Impact Parameter, Eikonal, Factorizing and Geometrical Scaling.

\section{REFERÊNCIAS BIBLIOGRÁFICAS}

1 - AKERLOF, C.W, et al. Hadron-proton Scattering at 50, 100 and 200 GeV/c Momentum. Phys. Rev., D14(11): $2864,1976$.

\section{2 - ALBERI, G. \& GOGG!, G. Diffraction of Subnuclear} Waves. Phys. Reports, 741(1):1 - 207, 1981.

3 - AMALDI, $U$. et al. The energy dependence of the proton-proton total cross-section for center-ofmass energies between 23 and $53 \mathrm{GeV}$. Phys. Lett.,
$448(1): 112,1973$.

4 - AMALDI, U. \& SCHUBERT, K.R. Impact parameter interpretation of proton-proton scattering from a critical review of all ISR data. Nucl. Phys., B166: $301-320,1980$.

5 - AMENDOLIA, S.R. et a]. Measurement of the total proton-proton cross - section at the ISR. Phys. Lett., $44 B(1): 119,1973$. 
6 - ASAD, Z. et al. Elastic scattering of charged mesons, antiprotons and protons and at incident momenta 20,30 and $50 \mathrm{GeV} / \mathrm{c}$ in the momentum transfer range 0.5 $\leq-\mathrm{t} \leq 8$ (GeVic) ${ }^{2}$. Nuct. Phys., B255: 273 - 327, 1985.

7 - AYRES, D.S. et al. $\pi^{ \pm}, K^{ \pm}, p p$ and $\overline{p p}$ elastic scottering from 50 to $175 \mathrm{GeV} / c$. Fhys. Rev., D15(11): 3105, 1985.

8 - BELLANDI, J.F'. et al. An attempt to extract semi-hard QCD effects from $\bar{p}$ p elastic scattering data. $\bar{z}$. Phys., C, 43: 651, 1989.

9 - BEVINGTON, L.A. Data reduction and error analysis for the physical sciences. New York: McGraw - Hill Book, 1969. p. 204-246.

10 - BURAS, A.J. \& DIAS DE DEUS, $\mathbf{J}$. Scaling law for the elastic differential cross section in pp scattering from geometrical scaling. Nucl. Phys., B71 : 481-492, 1974.

11 - CASTALDI, R. \& SANGUINETII, G. Elastic scattering and total cross section at very high energies. Ann. Rev. Nucl. Pant Sci., 35: 351-395, 1985.

12 - CHOU, T.T. Energy dependence of opaqueness of pp collisions at high energies. Found. Phys., 8(5,6): 319,1978

13 - CHOU, T.T. Impact parameter analysis of pp elastic scattering at $s^{12}=23$ and 62 GeV. Nucl. Phys., B139: 260-268, 1978 .

14 - CHOU, T.T. \& YANG, C.M. Model of elastic high - energy scattering. Phys. Rev., 17O(5): 2591, 1968.

15 - COVOLAN, R.J.M. et at. Impact parameter space analysisof kaon-proton elastic scattering. Hadranic J., 51, 1990.

16 - DIAS DE DEUS, J. Geometrical scaling, multiplicity distributions and cross sections. Nucl. Phys., B59: $231-236,1973$.

17 - FAJARDO, L.A. et al. Real part of the forward elastic. nuclear amplitude for $p p, \bar{p} p, \pi^{*} p, \pi^{-} p, K^{-} p$ and $K^{*} p$ scattering between 70 and 200 GeV/c. Phys. Rev., $024(1): 46,1981$.

18 - FEARNLEY, L.A. et at. impact parameter analysis of $\bar{p} p$ elastic scattering from $s^{n n}=7.6 \mathrm{GeV}$ to $546 \mathrm{GeV}$. CERN-EP(85 - 137): 1-78, 1985.

19 - FRANÇA, H.M. \& HAMA, Y. Energy dependence of the eikonal en pp elastic collisions. Phys. Rev. D19(11): $3261,1979$.

20 - GIACOMELLI, 6. Total cross sections and elastic scattering at high energies. Phys. Reports. 23(2): 123-235, 1976.

21 - GLAUBER, R.J. High energy collision theory. In: W.E. BRITIN et al. Lectures in Theoretical Physics. New York, 1959. Vol. I. p. 315-414.

22 - HAYOT, F. \& SUKHATME, U.P. Test of a generalized Chou-Yang ModeI at currently available high energies. Phys. Rev., D1O: 2183, 1974.

23 - KAMRAN, $M$, A review of elastic hadronic scattering at high energies and small momentum transfers. Phys. Reports. 108(5,6): 275-399, 1984.

24 - LIPKIN, H.J. et al. Two - component Pomeron and hadron total cross sections and real parts. Phys. Rev., D17: 366, 1978.

25 - MARTIN, A. Asymptotic behavior of the real part of the scattering amplitude at $-t \neq 0$. Lett. N. Cim. 7(16): 811, 1973.

26 - PÁDUA, A.B. Análise Fenomenológica no Canal -s do Espalhamento Elástico $\vec{p} \rho$ a Altas Enengias. Campinas, IFGW, 1989. Tese (Doutorado) UNICAMP.

27 - PERL, M.L. High Energy Hadron Physics. New York: John Wiley \& Sons, 1974.

28 - RUBINSTEIN, $R$, et al. Large momentum transfer elastic scattering of $\pi^{*}, K^{*}$ and $p^{*}$ on protons at 100 and $200 \mathrm{GeV} / \mathrm{c}$. Phys. Rev. D30: 1413, 1984. 